\title{
A new fuzzy fractional order model of transmission of Covid-19 with quarantine class
}

\author{
Asma Hanif ${ }^{1, \mathrm{a}}$, A. I. K. Butt ${ }^{1, \mathrm{~b}}$, Shabir Ahmad ${ }^{2, \mathrm{c}}$, Rahim Ud Din ${ }^{2, \mathrm{~d}}$, \\ Mustafa Inc In $^{3,4,5, \mathrm{e}}$ (iD \\ ${ }^{1}$ Department of Mathematics, Government College University Lahore, Lahore, Pakistan \\ 2 Department of Mathematics, University of Malakand, Dir(L), Khyber Pakhtunkhwa, Pakistan \\ 3 Department of Computer Engineering, Biruni University, Istanbul, Turkey \\ ${ }^{4}$ Department of Mathematics, Faculty of Science, Firat University, 23119 Elazig, Turkey \\ 5 Department of Medical Research, China Medical University, Taichung, Taiwan
}

Received: 20 September 2021 / Accepted: 13 November 2021

(C) The Author(s), under exclusive licence to Società Italiana di Fisica and Springer-Verlag GmbH Germany, part of Springer Nature 2021

\begin{abstract}
This paper is devoted to a study of the fuzzy fractional mathematical model reviewing the transmission dynamics of the infectious disease Covid-19. The proposed dynamical model consists of susceptible, exposed, symptomatic, asymptomatic, quarantine, hospitalized, and recovered compartments. In this study, we deal with the fuzzy fractional model defined in Caputo's sense. We show the positivity of state variables that all the state variables that represent different compartments of the model are positive. Using Gronwall inequality, we show that the solution of the model is bounded. Using the notion of the next-generation matrix, we find the basic reproduction number of the model. We demonstrate the local and global stability of the equilibrium point by using the concept of Castillo-Chavez and Lyapunov theory with the Lasalle invariant principle, respectively. We present the results that reveal the existence and uniqueness of the solution of the considered model through the fixed point theorem of Schauder and Banach. Using the fuzzy hybrid Laplace method, we acquire the approximate solution of the proposed model. The results are graphically presented via MATLAB-17.
\end{abstract}

\section{Introduction}

Covid-19 is a novel strain that has never been seen in humans before. The early signs of this disease include, cough, fever, and breathing problems. In the later stages of the disease, the patient may develop pneumonia, severe acute respiratory syndrome, renal failure, and possibly death. The first human case of Covid-19 was reported in Wuhan city, China. Afterward, it was discovered that the coronavirus spread rapidly from person to person throughout China.

\footnotetext{
a e-mail: asmahanifgcu@gmail.com

b e-mail: azhar.butt@gcu.edu.pk

c e-mail: shabirahmad2232@gmail.com

d e-mail: rahimmaths24@gmail.com

e e-mail: minc@firat.edu.tr (corresponding author)
} 
In addition, the infected cases were not restricted to the Chinese city of Wuhan; they also spread to other Chinese cities. Because the virus was not only found in China but also other parts of the world, it spread quickly. The behavior of the coronavirus indicates that it was once thought to be an epidemic illness. However, coronavirus cases spread across the world, and the sickness became a pandemic. Furthermore, it was shown that coronavirus symptoms in humans took 2 to 10 days to appear. To study different aspects of the model, many papers have published in the two years [1-3]. Recently, Dbouka and Drikakis connected fluid dynamics with epidemiology in [4]. They presented the vital relationship between weather seasonality, airborne virus transmission, and pandemic outbreaks over a whole year. Other important works of the same authors about transmission of diseases are listed in [5-8].

In this manuscript, we develop a mathematical model of the Covid-19 effect on society from a systemic perspective. With varied parameters, this mathematical model will be able to describe the rate of disease transmission, the influence of disease on the rate of susceptible cases, exposed cases, and infectious cases. Here, we examine model simulations and study the Covid-19 model for successful model outputs. To characterize the effect of Covid-19 disease, we have to consider the seven compartments: $S(t), E(t), I(t), A(t), Q(t), H(t), R(t)$. Let $S(t)$ represents susceptible population, $E(t)$ denotes the exposed population, $I(t)$ denotes the total number of infectious cases which have symptoms, and $A(t)$ infectious cases which have not symptoms, $Q(t)$ represents quarantined cases, $H(t)$ denotes the hospitalized cases and $R(t)$ represents the recovered population. The total population is $N(t)=S(t)+E(t)+$ $I(t)+A(t)+Q(t)+H(t)+R(t)$. The proposed integer order model is as follows:

$$
\left\{\begin{array}{l}
\mathrm{DS}(t)=\lambda-\left(\alpha_{1} I+\alpha_{2} A+\alpha_{3} H\right) S-\tau S, \\
\mathrm{DE}(t)=\left(\alpha_{1} I+\alpha_{2} A+\alpha_{3} H\right) S-\left(\kappa_{1}+\kappa_{2}+\tau\right) E, \\
\mathrm{DI}(t)=\kappa_{1} E+\sigma A-\left(\beta_{1}+\beta_{2}+\beta_{3}+\delta_{I}+\tau\right) I, \\
\mathrm{DA}(t)=\kappa_{2} E-\left(\gamma_{1}+\gamma_{2}+\sigma+\tau\right) A \\
\mathrm{DQ}(t)=\beta_{2} I+\gamma_{1} A-\left(v_{1}+v_{2}+\delta_{Q}+\tau\right) Q \\
\mathrm{DH}(t)=\beta_{1} I+v_{1} Q-\left(\vartheta+\delta_{H}+\tau\right) H \\
\mathrm{DR}(t)=\beta_{3} I+\gamma_{2} A+v_{2} Q+\vartheta H-\tau R .
\end{array}\right.
$$

where $\lambda$ represents the birth rate, and $\tau$ represents the death of infected people. Where individual moves from $S(t)$ to $E(t)$ after interacting with infected individuals with the transmission rate $\alpha_{1}, \alpha_{2}$, and $\alpha_{3}$. Between exposure and the onset of symptoms, Covid -19 has an incubation period of 2 to 14 days. After this period, exposed individual transits from the compartment $E(t)$ to either compartment $I(t)$ at the rate of $\kappa_{1}$ or compartment $A(t)$ at the rate of $\kappa_{2}$. An individual can move from the compartment $A(t)$ to $I(t)$ at the rate of $\sigma$, and also they can be quarantined at the rate $\gamma_{1}$ and can be recovered at the rate of $\gamma_{2}$ or dies naturally at the rate of $\tau$. Fig. 1

Quarantined people can also be hospitalized at the rate of $v_{1}$ and can also be recover from the virus $\nu_{2}$ or dies naturally at the rate of $\tau$ or can die with this disease at the rate of $\delta_{Q}$. Once an individual becomes infected coronavirus that individual develop immunity against the virus at the rate of $\beta_{3}$ or the individual will be hospitalized with a rate of $\beta_{1}$ or can be quarantined at the rate of $\beta_{2}$ or dies with the rate of $\tau$ or dies due to disease at the rate of $\delta_{I}$. When a person is admitted to the hospital, they are treated and develop immunity to the virus at a rate of $\vartheta$ or dies due to this Covid-19 disease at the rate of $\delta_{H}$.

Fractional differential equations (FDEs) have been applied to examine physical processes with higher precision and accuracy throughout the last few decades. Their uses may also be found in biology, physics, control theory, and other scientific and medical research disci- 


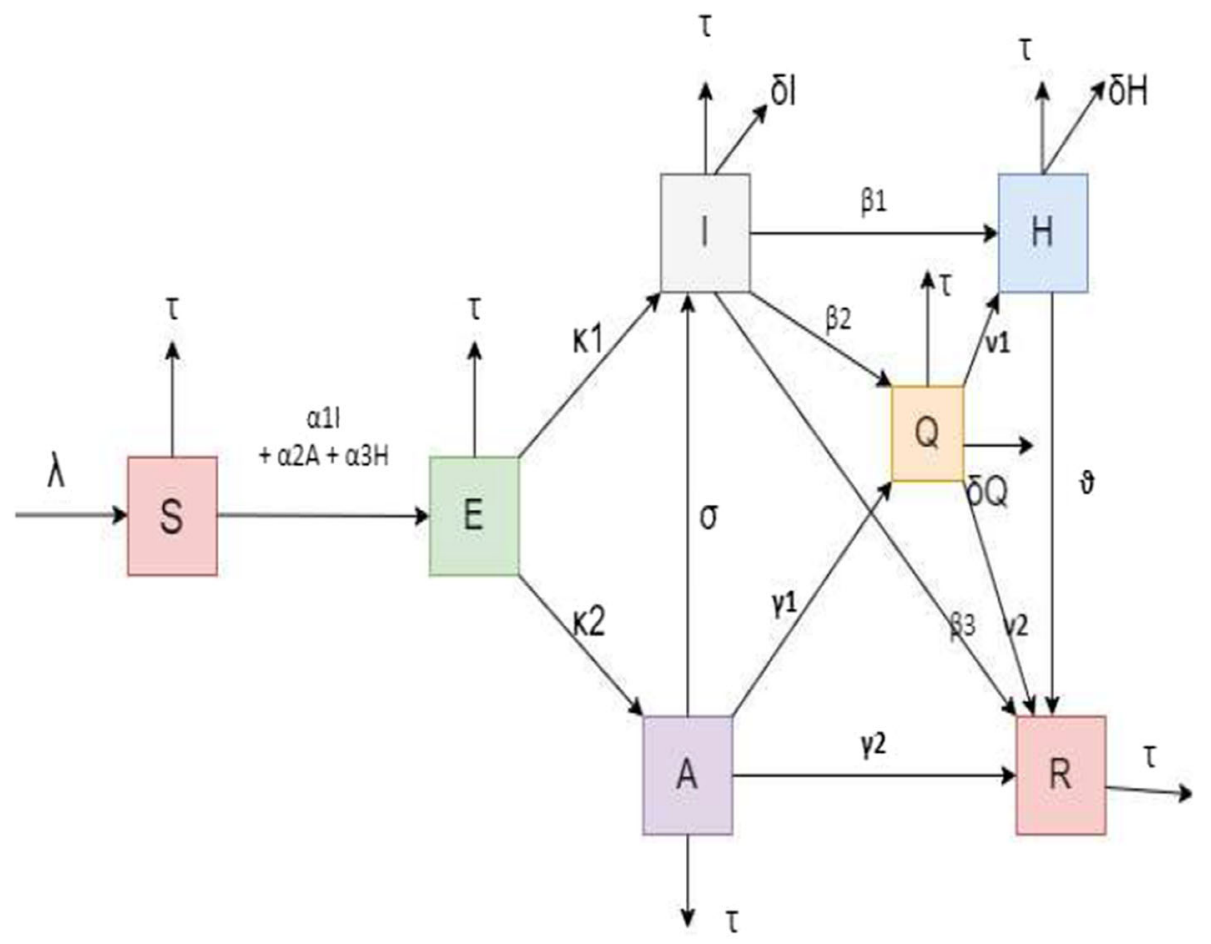

Fig. 1 Flow Diagram of Main Model

plines. The extension of classical integer-order calculus is modern calculus. FDEs are gaining popularity for simulating real-world situations due to their unique features that are not present in DEs. In contrast to DEs, FDEs are non-local and have memory effects, making them preferable to DEs. Moreover, in many situations, the model's future state is determined not just by its current state but also by its prior history, therefore FDEs have received a lot of attention from researchers in recent years. This is due to the fact that FDEs can more correctly explain the retention and heritable characteristics of diverse materials and processes than integer-order models [9-12]. We reformulate our mathematical model with Caputo fractional derivative for fractional order $y$ as:

$$
\left\{\begin{array}{l}
D_{t}^{y} S(t)=\lambda-\left(\alpha_{1} I+\alpha_{2} A+\alpha_{3} H\right) S-\tau S, \\
D_{t}^{y} E(t)=\left(\alpha_{1} I+\alpha_{2} A+\alpha_{3} H\right) S-\left(\kappa_{1}+\kappa_{2}+\tau\right) E, \\
D_{t}^{y} I(t)=\kappa_{1} E+\sigma A-\left(\beta_{1}+\beta_{2}+\beta_{3}+\delta_{I}+\tau\right) I, \\
D_{t}^{y} A(t)=\kappa_{2} E-\left(\gamma_{1}+\gamma_{2}+\sigma+\tau\right) A, \\
D_{t}^{y} Q(t)=\beta_{2} I+\gamma_{1} A-\left(v_{1}+v_{2}+\delta_{Q}+\tau\right) Q, \\
D_{t}^{y} H(t)=\beta_{1} I+v_{1} Q-\left(\vartheta+\delta_{H}+\tau\right) H, \\
D_{t}^{y} R(t)=\beta_{3} I+\gamma_{2} A+v_{2} Q+\vartheta H-\tau R .
\end{array}\right.
$$

FDEs or ODEs are used to study the mathematical models of different physical problems if the data or information are precise. When there is ambiguity or fuzziness in the information of a model, then fuzzy fractional operators are appropriate tools to model physical problems. For the first time, L.A. Zadeh has given the concept of the fuzzy set which is the generalization of 
the crisp set [13]. Later, the idea of fuzzy DEs and fuzzy FDEs was introduced [14-16]. The idea of fuzzy FDEs was used in many applied problems. For instance, Armand et al. studied the fractional relaxation oscillation DEs in a fuzzy concept [17]. Ahmad et al. analyzed fuzzy fractional Fisher's equation via fuzzy Laplace transform (FLT) [18]. In the literature [19], fuzzy dispersive PDE has been studied by using FLT. A fuzzy fractional mathematical model of COVID-19 has been investigated in [20]. Ahmad et al. investigated the transmission of COVID-19 under fuzzy fractional operator in Caputo sense [21]. Inspired by above work, we have taken model (2.1) under the fuzzy fractional Caputo derivative as:

$$
\left\{\begin{array}{l}
D_{t}^{y} S(t)=\tilde{\lambda}-\left(\tilde{\alpha_{1}} I+\tilde{\alpha_{2}} A+\tilde{\alpha_{2}} H\right) S-\tilde{\tau} S, \\
D_{t}^{y} E(t)=\left(\tilde{\alpha_{1}} I+\tilde{\alpha_{2}} A+\tilde{\alpha_{2}} H\right) S-\left(\tilde{\kappa_{1}}+\tilde{\kappa_{2}}+\tilde{\tau}\right) E, \\
D_{t}^{y} I(t)=\tilde{\kappa_{1}} E+\tilde{\sigma_{1}} A-\left(\tilde{\beta_{1}}+\tilde{\beta_{2}}+\tilde{\beta_{3}}+\tilde{\delta_{I}}+\tilde{\tau}\right) I, \\
D_{t}^{y} A(t)=\tilde{\kappa_{2}} E-\left(\tilde{\gamma_{1}}+\tilde{\gamma_{2}}+\tilde{\sigma}+\tilde{\tau}\right) A, \\
D_{t}^{y} Q(t)=\tilde{\beta_{2}} I+\tilde{\gamma_{1}} A-\left(\tilde{\nu_{1}}+\tilde{\nu_{2}}+\tilde{\delta} Q+\tilde{\tau}\right) Q, \\
D_{t}^{y} H(t)=\tilde{\beta}_{1} I+\tilde{\nu_{1}} Q-\left(\tilde{\vartheta}+\tilde{\delta}_{H}+\tilde{\tau}\right) H, \\
D_{t}^{y} R(t)=\tilde{\beta_{3}} I+\tilde{\gamma_{2}} A+\tilde{\nu_{2}} Q+\tilde{\vartheta} H-\tilde{\tau} R .
\end{array}\right.
$$

with fuzzy initial conditions, for $\zeta \in[0,1]$,

$$
\begin{aligned}
\tilde{S}(0, \zeta) & =(\underline{S}(0, \zeta), \bar{S}(0, \zeta)), \\
\tilde{E}(0, \zeta) & =(\underline{E}(0, \zeta), \bar{E}(0, \zeta)), \\
\tilde{I}(0, \zeta) & =(\underline{I}(0, \zeta), \bar{I}(0, \zeta)), \\
\tilde{A}(0, \zeta) & =(\underline{A}(0, \zeta), \bar{A}(0, \zeta)), \\
\tilde{Q}(0, \zeta) & =(\underline{Q}(0, \zeta), \bar{Q}(0, \zeta)), \\
\tilde{H}(0, \zeta) & =(\underline{H}(0, \zeta), \bar{H}(0, \zeta)), \\
\tilde{R}(0, \zeta) & =(\underline{R}(0, \zeta), \bar{R}(0, \zeta)) .
\end{aligned}
$$

\section{Fundamental results}

In this part, we list some fundamental concepts of fuzzy set. All the definitions and other related materials are taken from [22-24].

Definition 2.1 ([22]) A fuzzy set $\omega: \mathbb{R} \rightarrow[0,1]$ is said to fuzzy number if:

(i) $\omega$ is upper semi continuous.

(ii) $\omega$ is convex, i.e., $\forall f, g \in \mathbb{R}$ and $\hbar \in[0,1],,(\omega(\hbar f+(1-\hbar) g)) \geq \min \{\omega(f), \omega(g)\}$ holds.

(iii) $\omega$ is normal, i.e., $\exists x_{o} \in \mathbb{R}$ such that $\omega\left(x_{o}\right)=1$.

(iv) The closure of ( $\operatorname{supp} \omega$ ) is compact, where supp $\omega=\{x \in R \mid \omega(x)>0\}$ represents the support of $\omega$.

Let $G$ denotes the set of all fuzzy numbers.

Definition 2.2 ([23,24]) Let $\omega \in G$. For any $\zeta \in(0,1]$, the $\zeta$-level set of $\omega$, represented by $\omega^{\zeta}$, is defined as: $\omega^{\zeta}=\{z \in R \mid \omega(z) \geq \zeta\}$.

Definition 2.3 ([24]) Let $\omega \in G$, then the parametric form of $\omega$ is denoted by $[\underline{\omega}(\zeta), \bar{\omega}(\zeta)]$, where $0 \leq \zeta \leq 1$, which satisfies: 
(i) $\underline{\omega}(\zeta)$ is left continuous on $(0,1]$, non-decreasing and right continuous at 0 .

(ii) $\overline{\bar{\omega}}(\zeta)$ is right continuous on $(0,1]$ and non-increasing and right continuous at 0 .

(iii) $\underline{\omega}(\zeta) \leq \bar{\omega}(\zeta)$.

Definition 2.4 ([24]) Consider a mapping $\psi: G \times G \longrightarrow \mathbb{R}$ and let $r, s \in G$, such that $r=(\underline{r}(\zeta), \bar{r}(\zeta))$ and $s=(\underline{s}(\zeta), \bar{s}(\zeta))$. The Hausdorff distance between $r$ and $s$ is given by

$$
\psi(r, s)=\sup _{\zeta \in[0,1]}[\max (|\underline{r}(\zeta)-\underline{s}(\zeta)|,|\bar{r}(\zeta)-\bar{s}(\zeta)|)] .
$$

In $G$, the metric $\psi$ satisfies:

(i) $\psi(r+t, s+t)=\psi(r, s) \forall r, s, t \in G$.

(ii) $\psi(r \sigma, s \sigma)=|\sigma| \psi(r, s) \forall r, s \in G, \sigma \in \mathbb{R}$.

(iii) $\psi(r+\xi, s+\theta) \leq \psi(r, s)+\psi(\xi, \theta) \forall r, s, \xi, \theta \in G$.

(iv) $(G, \psi)$ is a complete metric space.

Definition 2.5 ([24]) Let $\varepsilon_{1}, \varepsilon_{2} \in G$. If $\exists \varepsilon_{3} \in G$ such that $\varepsilon_{1}=\varepsilon_{2}+\varepsilon_{3}$, then $\varepsilon_{3}$ is said to be $H$-difference of $\varepsilon_{1}$ and $\varepsilon_{2}$, denoted by $\varepsilon_{1}-\varepsilon_{2}$.

Definition 2.6 ([24]) Let $\Theta: \mathbb{R} \rightarrow G$ be a fuzzy function, then $\Theta$ is said to be fuzzy continuous if for any $\epsilon>0 \exists \delta>0$ and a fixed value of $\lambda_{o} \in\left[\zeta_{1}, \zeta_{2}\right]$, we have $\rho\left(\Theta(\lambda), \Theta\left(\lambda_{o}\right)\right)<\epsilon$ whenever $\left|\lambda-\lambda_{0}\right|<\delta$.

Definition 2.7 ([24]) Let $\psi$ be a continuous fuzzy function on $[0, d] \subseteq \mathbb{R}$, a FFI in ReimannLioville sense is defined as

$$
I^{y} \psi(t)=\frac{1}{\Gamma(y)} \int_{0}^{t}(t-\mu)^{y-1} \psi(\mu) \mathrm{d} \mu,
$$

where $y \in(0,1)$ further, if $\psi \in C^{F}[0, d] \cap L^{F}[0, d]$, where $C^{F}[0, d]$ and $L^{F}[0, d]$ are the spaces of fuzzy continuous functions and fuzzy Lebesgue integrable function, respectively, then FFI is given by

$$
\left[I^{y} \psi(t)\right]_{\zeta}=\left(I^{y} \underline{\psi}_{\zeta}(t), I^{y} \bar{\psi}_{\zeta}(t)\right), 0 \leq \zeta \leq 1
$$

where

$$
\begin{aligned}
I^{y} \underline{\psi}_{\zeta}(t) & =\frac{1}{\Gamma(y)} \int_{0}^{t}(t-\mu)^{y-1} \underline{\psi}_{\zeta}(t) \operatorname{Dir}(L) \mathrm{d} \mu, \\
I^{y} \bar{\psi}_{\zeta}(t) & =\frac{1}{\Gamma(y)} \int_{0}^{t}(t-\mu)^{y-1} \bar{\psi}_{\zeta}(t) \mathrm{d} \mu .
\end{aligned}
$$

Definition 2.8 ([24]) If a fuzzy function $\psi \in C^{F}[0, d] \cap L^{F}[0, d]$ is such that $\psi=$ $\left(\underline{\psi}_{\zeta}(t), \bar{\psi}_{\zeta}(t)\right), 0 \leq \zeta \leq 1$ and $t_{1} \in(0, d)$, then the fuzzy fractional Caputo derivative is given by

$$
\left[D^{y} \psi\left(t_{0}\right)\right]_{\zeta}=\left(D^{y} \underline{\psi}_{\zeta}\left(t_{0}\right), D^{y} \bar{\psi}_{\zeta}\left(t_{0}\right)\right), 0 \leq y \leq 1,
$$

where

$$
D^{y} \underline{\psi}_{\zeta}\left(t_{0}\right)=\frac{1}{\Gamma(n-y)}\left[\int_{0}^{t}(t-\xi)^{n-y-1} \frac{\mathrm{d}^{n}}{\mathrm{~d} \xi^{n}} \psi_{\zeta}(\xi) \mathrm{d} \xi\right]_{t=t_{0}},
$$




$$
D^{y} \bar{\phi}_{\zeta}\left(t_{0}\right)=\frac{1}{\Gamma(n-y)}\left[\int_{0}^{t}(t-\xi)^{n-y-1} \frac{\mathrm{d}^{n}}{\mathrm{~d} \xi^{n}} \bar{\phi}_{\zeta}(\xi) \mathrm{d} \xi\right]_{t=t_{0}},
$$

where $n=\lceil y\rceil$.

Definition 2.9 ([24]) Let $\psi$ be a continuous fuzzy-valued function. Assume that $\psi(\mathcal{X}) e^{-s \mathcal{X}}$ is improper fuzzy Riemann-integral on $[0, \infty)$, then its fuzzy Laplace transform is represented by

$$
\mathcal{L}[\psi(\mathcal{X})]=\int_{0}^{\infty} \psi(\mathcal{X}) \cdot e^{-s \mathcal{X}} \mathrm{d} \mathcal{X}
$$

For $0 \leq \zeta \leq 1$, the parametric form of $\psi(\mathcal{X})$ is represented by

$$
\int_{0}^{\infty} \psi(\mathcal{X}, \zeta) \cdot e^{-s \mathcal{X}} \mathrm{d} \mathcal{X}=\left(\int_{0}^{\infty} \underline{\psi}(\mathcal{X}, \zeta) \cdot e^{-s \mathcal{X}} \mathrm{d} \mathcal{X}, \int_{0}^{\infty} \bar{\psi}(\mathcal{X}, \zeta) \cdot e^{-s \mathcal{X}} \mathrm{d} \mathcal{X}\right) .
$$

Hence,

$$
\mathcal{L}[\psi(\mathcal{X}, \zeta)]=(\mathcal{L} \underline{\psi}(\mathcal{X}, \zeta), \mathcal{L} \bar{\psi}(\mathcal{X}, \zeta))
$$

Definition 2.10 ([24]) The Laplace transform (LT) of $D^{y} \psi(t)$ is given by

$$
\mathcal{L}\left[D^{y} \psi(t)\right]=s^{y} \mathcal{L}[\psi(t)]-s^{y-1}[\psi(0)] .
$$

\section{Qualitative aspects of the model (1)}

In this section, we now describe the some qualities of our model to show that it is more realistic and feasible. For this purpose, we have proved the following properties of the solutions of the model (1.1).

\subsection{Positivity of solution}

Let $u=(S(t), E(t), I(t), A(t), Q(t), H(t), R(t))$, the following shows that $u$ is nonnegative $\forall t \geq 0$.

Theorem $1 \forall t \geq 0$, the solution $u(t)$ of the system (1.1) is non negative, if all ICs are non-negative.

Proof Since $u(0) \geq 0$. Consider the first compartment $S(t)$

$$
D(S(t))+\left[\left(\alpha_{1} I+\alpha_{2} A+\alpha_{3} H\right)+\tau\right] S=\lambda .
$$

The above equation can be written as

$$
D(S(t))+(\chi+\tau) S=\lambda,
$$


where $\alpha_{1} I+\alpha_{2} A+\alpha_{3} H=\chi$, now multiplying the above equation by $e^{\left(\tau t+\int_{0}^{t} \chi(v) \mathrm{d} v\right)}$, we have

$$
\frac{\mathrm{d}}{\mathrm{d} t}\left[e^{\left(\tau t+\int_{0}^{t} \chi(v) \mathrm{d} v\right)} S\right]=\lambda e^{\left(\tau z+\int_{0}^{t} \chi(v) \mathrm{d} v\right)} .
$$

Integrating both sides over the interval $[0, \bar{t}]$, where $\bar{t}=\sup \{t>0 ; u(t)>0\}$,

$$
\begin{array}{r}
S(\bar{t})\left[e^{\left(\tau \bar{t}+\int_{0}^{t} \chi(v) d v\right)}\right]-S(0)=\lambda \int_{0}^{t} e^{\left(\tau t+\int_{0}^{t} \chi(v) \mathrm{d} v\right)} \mathrm{d} t \\
S(\bar{t})=\left[e^{-\left(\tau \bar{t}+\int_{0}^{t} \chi(v) d v\right)}\right]+S(0)+\lambda \int_{0}^{t} e^{\left(\tau t+\int_{0}^{t} \chi(v) d v\right)} \mathrm{d} t,
\end{array}
$$

which shows that $S(\bar{t})>0$. On similar fashion, the positivity of the remaining state variables can be proved.

\subsection{Boundedness of solution}

Here, we show that $u(t)$ is bounded with help of Gronwall inequality.

Theorem 2 The solutions of $u(t)$ of the system (1.1) are bounded.

Proof Consider the total population $N(t)$ as:

$$
N(t)=S(t)+E(t)+I(t)+A(t)+Q(t)+H(t)+R(t) .
$$

Now, from the above equation and the equations of the model (1.1), we have

$$
D N(t)=\lambda-\delta_{y}-\delta_{q}-\delta_{h}-\tau N .
$$

At $t=0, N(t)$ is given by

$$
N(0)=S(0)+E(0)+I(0)+A(0)+Q(0)+H(0)+R(0) .
$$

Let for any IC

$$
N(0) \leq \frac{\lambda}{\tau}
$$

Now,

$$
D N(t) \leq \lambda-\tau N
$$

Consider an ODE as:

$$
D X(t)=q-p X .
$$

The solution of the above ODE is as follows

$$
X(t)=\frac{q}{p}+\left(X(0)-\frac{q}{p}\right) e^{-p z}
$$

where $\mathrm{a}$ and $\mathrm{b}$ are constants. therefore by Gronwall's inequality, we get

$$
N(t) \leq \frac{\lambda}{\tau}+\left(N(0)-\frac{\lambda}{\tau}\right) e^{-\tau t}
$$


thus, we conclude that

$$
N(t) \leq \frac{\lambda}{\tau},
$$

so above equation provided bounds for $t \geq 0$. Hence,

$$
\lim _{t \rightarrow \infty} N(t) \leq \frac{\lambda}{\tau}
$$

\subsection{Disease free equilibrium point (DFE)}

For the proposed model (1.1), the DFE $P_{o}$ can be obtained by putting the all equations equals to zero. Consider

$$
\left\{\begin{array}{l}
0=\lambda-\left(\alpha_{1} I+\alpha_{2} A+\alpha_{3} H\right) S-\tau S, \\
0=\left(\alpha_{1} I+\alpha_{2} A+\alpha_{3} H\right) S-\left(\kappa_{1}+\kappa_{2}+\tau\right) E, \\
0=\kappa_{1} E+\sigma A-\left(\beta_{1}+\beta_{2}+\beta_{3}+\delta_{I}+\tau\right) I, \\
0=\kappa_{2} E-\left(\gamma_{1}+\gamma_{2}+\sigma+\tau\right) A, \\
0=\beta_{2} I+\gamma_{1} A-\left(v_{1}+v_{2}+\delta_{Q}+\tau\right) Q, \\
0=\beta_{1} I+v_{1} Q-\left(\vartheta+\delta_{H}+\tau\right) H, \\
0=\beta_{3} I+\gamma_{2} A+v_{2} Q+\vartheta H-\tau R .
\end{array}\right.
$$

From all above equation, we have

$$
P_{o}=\left(\frac{\lambda}{\tau}, 0,0,0,0,0\right) .
$$

3.4 Endemic equilibrium point(EEP)

We assume that infected persons remain in the community for the endemic equilibrium point (EEP). As a result, $I \neq 0$ and $E \neq 0$ are equal in this case. The EEP $P^{*}$ is given by

$$
P^{*}=\left(S^{*}, E^{*}, I^{*}, A^{*}, Q^{*}, H^{*}, R^{*}\right),
$$

where

$$
\begin{aligned}
S^{*} & =\frac{\lambda}{C_{1}+\tau} \\
E^{*} & =\frac{\lambda C_{1}}{L_{1}\left(C_{1}+\tau\right)}, \\
I^{*} & =\frac{\kappa_{1} L_{3}+\sigma \kappa_{2}}{L_{2} L 3}\left(\frac{\lambda C_{1}}{L_{1}\left(C_{1}+\tau\right)}\right) \\
A^{*} & =\frac{\kappa_{2}}{L_{3}}\left(\frac{\lambda C_{1}}{L_{1}\left(C_{1}+\tau\right)}\right) \\
Q^{*} & =\frac{\beta_{2} \kappa_{1} L_{3}+\sigma \kappa_{2} \beta_{2}+\gamma_{1} \kappa_{2} L_{2}}{L_{2} L_{3} L_{4}}\left(\frac{\lambda C_{1}}{L_{1}\left(C_{1}+\tau\right)}\right) \\
H^{*} & =\frac{\beta_{1} L_{4} L_{3} \kappa_{1}+\sigma \kappa_{2} \beta_{1} L_{4}+v_{1} \beta_{2} \kappa_{1} L_{3}+v_{1} \kappa_{2} \beta_{2} \sigma+\gamma_{1} \kappa_{2} L_{2} v_{1}}{L_{2} L_{3} L_{4} L_{5}}\left(\frac{\lambda C_{1}}{L_{1}\left(C_{1}+\tau\right)}\right) \\
R^{*} & =\frac{1}{\tau}\left(\beta_{3} I^{*}+\gamma_{2} A^{*}+v_{2} Q^{*}\right)
\end{aligned}
$$


where

$$
\begin{aligned}
& C_{1}=\alpha_{1} I+\alpha_{2} A+\alpha_{3} H, \\
& L_{1}=\kappa_{1}+\kappa_{2}+\tau, \\
& L_{2}=\beta_{1}+\beta_{2}+\beta_{3}+\delta_{I}+\tau, \\
& L_{3}=\gamma_{1}+\gamma_{2}+\sigma+\tau, \\
& L_{4}=v_{1}+\nu_{2}+\delta_{Q}+\tau, \\
& L_{5}=\vartheta+\delta_{H}+\tau .
\end{aligned}
$$

\subsection{Reproduction number}

The reproduction number (RN) is a mathematical quantity that defines whether or not an infectious illness is spreading in the community. It also offers a criterion for DFE stability. In most cases, we use the next generation matrix technique to $\mathrm{RN}$ for a model. Consider

$$
\left\{\begin{array}{l}
\mathrm{DE}(t)=\left(\alpha_{1} I+\alpha_{2} A+\alpha_{3} H\right) S-\left(\kappa_{1}+\kappa_{2}+\tau\right) E, \\
\mathrm{DI}(t)=\kappa_{1} E+\sigma A-\left(\beta_{1}+\beta_{2}+\beta_{3}+\delta_{I}+\tau\right) I, \\
\mathrm{DA}(t)=\kappa_{2} E-\left(\gamma_{1}+\gamma_{2}+\sigma+\tau\right) A, \\
\mathrm{DQ}(t)=\beta_{2} I+\gamma_{1} A-\left(v_{1}+v_{2}+\delta_{Q}+\tau\right) Q, \\
\mathrm{DH}(t)=\beta_{1} I+v_{1} Q-\left(\vartheta+\delta_{H}+\tau\right) H .
\end{array}\right.
$$

The column matrix $\mathcal{F}$ representing the rate of new infection arrival is expressed as

$$
\mathcal{F}=\left(\begin{array}{c}
\left(\alpha_{1} I+\alpha_{2} A+\alpha_{3} H\right) S \\
0 \\
0 \\
0 \\
0
\end{array}\right)=\left(\begin{array}{c}
\left(\alpha_{1} I+\alpha_{2} A+\alpha_{3} H\right) \frac{\lambda}{\tau} \\
0 \\
0 \\
0 \\
0
\end{array}\right),
$$

and the column matrix $\mathcal{V}$ of transitional elements in the compartments under consideration is

$$
\mathcal{V}=\left(\begin{array}{c}
\left(\kappa_{1}+\kappa_{2}+\tau\right) E \\
-\kappa_{1} E-\sigma A+\left(\beta_{1}+\beta_{2}+\beta_{3}+\delta_{I}+\tau\right) I \\
-\kappa_{2} E+\left(\gamma_{1}+\gamma_{2}+\sigma+\tau\right) A \\
-\beta_{2} I-\gamma_{1} A+\left(\nu_{1}+\nu_{2}+\delta_{Q}+\tau\right) Q \\
-\beta_{1} I-v_{1} Q+\left(\vartheta+\delta_{H}+\tau\right) H
\end{array}\right)
$$


For the considered model, the Jacobian Matrix $\mathcal{F}$ is defined as

$$
\begin{aligned}
& \mathcal{F}=\left(\frac{\partial \mathcal{F}_{k}}{\partial x_{k}}\right)_{P_{\mathrm{DFE}}}, k=1,2,3,4,5 . \\
& \mathcal{F}=\left(\begin{array}{cccccc}
0 & \alpha_{1} \frac{\lambda}{\tau} & \alpha_{2} \frac{\lambda}{\tau} & 0 & \alpha_{2} \frac{\lambda}{\tau} \\
0 & 0 & 0 & 0 & 0 \\
0 & 0 & 0 & 0 & 0 \\
0 & 0 & 0 & 0 & 0 \\
0 & 0 & 0 & 0 & 0
\end{array}\right)=\left(\begin{array}{ccccc}
0 & A_{1} & A_{2} & 0 & A_{3} \\
0 & 0 & 0 & 0 & 0 \\
0 & 0 & 0 & 0 & 0 \\
0 & 0 & 0 & 0 & 0 \\
0 & 0 & 0 & 0 & 0
\end{array}\right) .
\end{aligned}
$$

Similarly, the Jacobian matrix for $\mathcal{V}$ is defined as

$$
\mathcal{V}=\left(\frac{\partial \mathcal{V}_{k}}{\partial x_{k}}\right)_{P_{\mathrm{DFE}}}, k=1,2,3,4,5
$$

After some steps, we get

$$
\mathcal{V}=\left(\begin{array}{ccccc}
\left(\kappa_{1}+\kappa_{2}+\tau\right) & 0 & 0 & 0 & 0 \\
-\kappa_{1} & \left(\beta_{1}+\beta_{2}+\beta_{3}+\delta_{I}+\tau\right) & -\sigma & 0 & 0 \\
-\kappa_{2} & 0 & \left(\gamma_{1}+\gamma_{2}+\sigma+\tau\right) & 0 & 0 \\
0 & -\beta_{2} & -\gamma_{1} & \left(\nu_{1}+\nu_{2}+\delta_{Q}+\tau\right) & 0 \\
0 & -\beta_{1} & 0 & -\nu_{1} & \left(\vartheta+\delta_{H}+\tau\right)
\end{array}\right)
$$

The above matrix can be written as

$$
\mathcal{V}=\left(\begin{array}{ccccc}
L_{1} & 0 & 0 & 0 & 0 \\
-\kappa_{1} & L_{2} & -\sigma & 0 & 0 \\
-\kappa_{2} & 0 & L_{3} & 0 & 0 \\
0 & -\beta_{2} & -\gamma_{1} & L_{4} & 0 \\
0 & -\beta_{1} & 0 & -v_{1} & L_{5}
\end{array}\right)
$$

Inverse of the matrix $V$ is computed, and we get

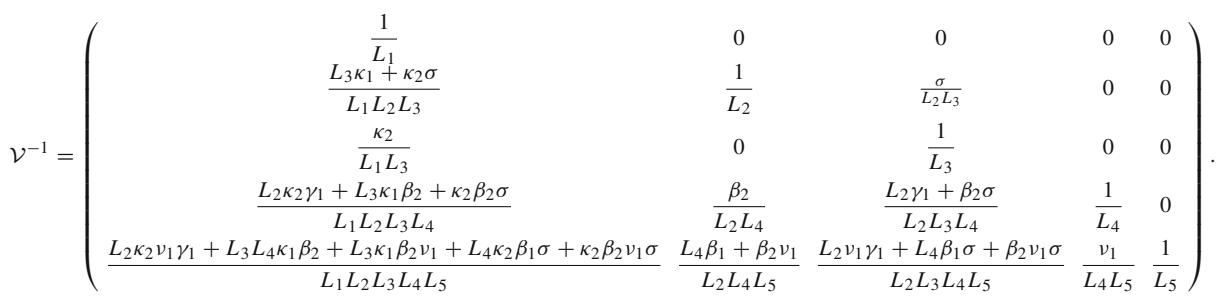

Since $R_{O}=$ maximum eigenvalue of the matrix $\mathcal{F} \mathcal{V}^{-1}$, therefore $R_{O}=$

$\underline{A_{1} L_{3} L_{4} L_{5} \kappa_{1}+A_{1} L_{4} L_{5} \kappa_{2} \sigma+A_{2} L_{2} L_{4} L_{5} \kappa_{2}+A_{3} L_{2} \gamma_{1} \kappa_{2} \nu_{1}+A_{3} L_{3} L_{4} \beta_{1} \kappa_{1}+A_{3} L_{3} \beta_{2} \kappa_{1} \nu_{1}+A_{3} L_{4} \beta_{1} \kappa_{2} \sigma+A_{3} \beta_{2} \kappa_{2} \nu_{1} \sigma}$

where

$$
A_{1}=\alpha_{1}\left(\frac{\lambda}{\tau}\right), A_{2}=\alpha_{2}\left(\frac{\lambda}{\tau}\right), A_{3}=\alpha_{3}\left(\frac{\lambda}{\tau}\right)
$$

and

$$
\begin{aligned}
& L_{1}=\kappa_{1}+\kappa_{2}+\tau, \quad L_{2}=\beta_{1}+\beta_{2}+\beta_{3}+\delta_{I}+\tau, \quad L_{3}=\gamma_{1}+\gamma_{2}+\sigma+\tau, \\
& L_{4}=v_{1}+v_{2}+\delta_{Q}+\tau, \quad L_{5}=\vartheta+\delta_{H}+\tau .
\end{aligned}
$$


3.6 Stability of equilibrium points

In this part, the local asymptotically stability (LAS) and global asymptotically stability (GLS) of the model (1.1) around the DFE and EE points are presented. The Jacobian matrix at DFE point is given as

$$
\mathcal{J}=\left(\begin{array}{ccccccc}
-\tau & 0 & -\alpha_{1}\left(\frac{\lambda}{\tau}\right) & -\alpha_{2}\left(\frac{\lambda}{\tau}\right) & 0 & -\alpha_{1}\left(\frac{\lambda}{\tau}\right) & 0 \\
0 & -L_{1} & \alpha_{1}\left(\frac{\lambda}{\tau}\right) & \alpha_{1}\left(\frac{\lambda}{\tau}\right) & 0 & \alpha_{1}\left(\frac{\lambda}{\tau}\right) & 0 \\
0 & \kappa_{1} & -L_{2} & \sigma & 0 & 0 & 0 \\
0 & \kappa_{2} & 0 & -L_{3} & 0 & 0 & 0 \\
0 & 0 & \beta_{2} & \gamma_{1} & -L_{4} & 0 & 0 \\
0 & 0 & \beta_{1} & 0 & \nu_{1} & -L_{5} & 0 \\
0 & 0 & \beta_{3} & \gamma_{2} & \nu_{2} & \vartheta & -\tau
\end{array}\right),
$$

which can be written as

$$
\mathcal{J}=\left(\begin{array}{ccccccc}
-\tau & 0 & -A_{1} & -A_{2} & 0 & -A_{3} & 0 \\
0 & -L_{1} & A_{1} & A_{2} & 0 & A_{3} & 0 \\
0 & \kappa_{1} & -L_{2} & \sigma & 0 & 0 & 0 \\
0 & \kappa_{2} & 0 & -L_{3} & 0 & 0 & 0 \\
0 & 0 & \beta_{2} & \gamma_{1} & -L_{4} & 0 & 0 \\
0 & 0 & \beta_{1} & 0 & \nu_{1} & -L_{5} & 0 \\
0 & 0 & \beta_{3} & \gamma_{2} & \nu_{2} & \vartheta & -\tau
\end{array}\right) .
$$

Now, we are in position to present LAS of DFE and EE points. The following theorems demonstrate the LAS of the model (1.1) at DFE and EE points.

Theorem 3 If $R_{o}<1$, the model (1.1) is LAS at DFE point, otherwise unstable for $R_{O}>1$.

Proof First, we need to find eigenvalues of the above Jacobian matrix. With the help maple, we get

$$
\begin{aligned}
& \lambda_{1}=-L_{4}, \lambda_{2}=-L_{1}, \lambda_{3}=\frac{A_{1} \kappa_{1}-L_{1} L_{2}}{L_{1}}, \\
& \lambda_{4}=-\frac{A_{1} L_{3} \kappa_{1}+A_{1} \kappa_{2} \sigma+A_{2} L_{2} \kappa_{2}-L_{1} L_{1} L_{3}}{A_{1} \kappa_{1}-L_{1} L_{2}},
\end{aligned}
$$

$\lambda_{5}=-\frac{A_{1} L_{3} L_{4} L_{5} \kappa_{1}+A_{1} L_{4} L_{5} \kappa_{2} \sigma+A_{2} L_{2} L_{4} L_{5} \kappa_{2}+A_{3} L_{3} L_{4} \beta_{1} \kappa_{1}+A_{3} L_{4} \beta_{1} \kappa_{2} \sigma-L_{1} L_{2} L_{3} L_{4} L_{5}+A_{3} L_{2} \gamma_{1} \kappa_{2} \nu_{1}+A_{3} L_{3} \beta_{2} \kappa_{1} \nu_{1}+A_{3} \beta_{2} \kappa_{2} \nu_{1} \sigma}{L_{4}\left(A_{1} L_{3} \kappa_{1}+A_{1} \kappa_{2} \sigma+A_{2} L_{2} \kappa_{2}-L_{1} L_{2} L_{3}\right)}$, $\lambda_{6}=-\tau, \lambda_{7}=-\tau$,

as we see that $\lambda_{1}=-L_{4}, \lambda_{2}=-L_{1}, \lambda_{5}=-\tau, \lambda_{5}=-\tau$ all are negative. Now, $\lambda_{3}=$ $L_{2}\left(R_{o}-P-L_{2}\right), \lambda_{3}=L_{2} Q_{1}$. When $R_{o}<1 ; Q_{1}<0$, and $L_{2}>0$, then $\lambda_{3}<0$, where

$$
\begin{aligned}
P= & \frac{\alpha_{2} \kappa_{2} \lambda}{L_{1} L_{3} \tau}+\frac{\alpha_{3} \beta_{1} \kappa_{1} \lambda}{L_{1} L_{2} L_{5} \tau}+\frac{\alpha_{1} \kappa_{2} \lambda \sigma}{L_{1} L_{2} L_{3}}+\frac{\alpha_{3} \beta_{2} \kappa_{1} \lambda \nu_{1}}{L_{1} L_{2} L_{4} L_{5} \tau}+\frac{\alpha_{3} \beta_{1} \kappa_{2} \lambda \sigma}{L_{1} L_{2} L_{3} L_{5} \tau} \\
& +\frac{\alpha_{3} \gamma_{1} \kappa_{2} \lambda v_{1}}{L_{1} L_{3} L_{4} L_{5} \tau}+\frac{\alpha_{3} \beta_{2} \lambda \kappa_{2} \nu_{1} \sigma}{L_{1} L_{2} L_{4} L_{3} L_{5} \tau},
\end{aligned}
$$

and $\lambda_{4}=-\frac{L_{3}\left(R_{o}-M-1\right)}{R_{o}-P}$. If $R_{O}<1$, consider the numerator $R_{O}-M-1<0$, so numerator is positive and consider the denominator $R_{O}-P<0$, when $R_{O}<1$, then $\lambda_{4}<0$, where

$$
M=\frac{\alpha_{3} \beta_{1} \kappa_{1} \lambda}{L_{1} L_{2} L_{5} \tau}+\frac{\alpha_{3} \beta_{2} \kappa_{1} \lambda \nu_{1}}{L_{1} L_{2} L_{4} L_{5} \tau}+\frac{\alpha_{3} \beta_{3} \kappa_{2} \lambda \sigma}{L_{1} L_{2} L_{3} L_{5} \tau}+\frac{\alpha_{3} \gamma_{1} \kappa_{2} \lambda v_{1}}{L_{1} L_{3} L_{4} L_{5} \tau}+\frac{\alpha_{3} \beta_{2} \kappa_{2} \nu_{1} \lambda \sigma}{L_{1} L_{2} L_{3} L_{4} L_{5} \tau},
$$


also for $\lambda_{5}=-\frac{L_{5}\left(R_{o}-1\right)}{\left(R_{o}-M\right)}$ and $R_{o}<1$, the numerator $\left(R_{o}-1\right)<0$ and $L_{5}>0$. So $-\left(L_{5}\left(R_{o}-1\right)\right)>0$ and for denominator $R_{o}-M<0$, the eigenvalue $\lambda_{3}<0$. It demonstrates that when $R_{O}<1$, all eigenvalues are negative, but not when $R_{O}>1$. Model (1.1) is therefore proven to be locally LAS for $R_{O}<1$.

Theorem 4 There is a unique EEP $\left(S^{*}, E^{*}, I^{*}, A^{*}, Q^{*}, H^{*}, R^{*}\right)$ of system (1.1) under condition $C_{2} C_{5}-\beta_{2} \gamma_{1}^{2}>0$ and $\alpha_{1} k_{1} C_{2}-\alpha_{2} k_{2} C_{1}>0$.

Proof The Jacobian matrix of model (1.1) at $\left(S^{*}, E^{*}, I^{*}, A^{*}, Q^{*}, H^{*}, R^{*}\right)$ is

$$
M=\left(\begin{array}{ccccccc}
-C_{6}-\tau & 0 & \alpha_{1} & \alpha_{2} & 0 & \alpha_{2} & 0 \\
C_{6} & -C_{3} & \alpha_{1} & \alpha_{2} & 0 & \alpha_{2} & 0 \\
0 & \kappa_{1} & -C_{1} & \sigma & 0 & 0 & 0 \\
0 & \kappa_{2} & 0 & -C_{2} & 0 & 0 & 0 \\
0 & 0 & \beta_{2} & \gamma_{1} & -C_{5} & 0 & 0 \\
0 & 0 & \beta_{1} & 0 & \nu_{1} & -C_{4} & 0 \\
0 & 0 & \beta_{3} & \gamma_{2} & \nu_{2} & \vartheta & -\tau
\end{array}\right),
$$

where

$$
\left\{\begin{array}{l}
C_{1}=\beta_{1}+\beta_{2}+\beta_{3}+\delta_{I}+\tau, \\
C_{2}=\gamma_{1}+\gamma_{2}+\sigma+\tau, \\
C_{3}=\kappa_{1}+\kappa_{2}+\tau \\
C_{4}=\vartheta+\delta_{H}+\tau \\
C_{5}=v_{1}+\nu_{2}+\delta_{Q}+\tau \\
C_{6}=\alpha_{1} I^{*}+\alpha_{2} A^{*}+\alpha_{3} H^{*}
\end{array}\right.
$$

We know that the stability of $\left(S^{*}, E^{*}, I^{*}, A^{*}, Q^{*}, H^{*}, R^{*}\right)$ is determine by $\operatorname{tr}(M)$.

$$
\operatorname{tr}(M)=-C_{6}-C_{3}-C_{1}-C_{2}-C_{5}-C_{4}-2 \tau<0 .
$$

From the continuity of $\operatorname{tr}(M)$ on the variables, we get that $\operatorname{tr}(M)<0$. This completes our conclusion.

\subsubsection{Global stability}

The global stability of the model (1.1) at both equilibrium points is examined in this subsection. We use Castillo-Chavez's method for global stability at the DFE point and Lyapunov's theory with the LaSalle invariant principle for global stability at the EE point.

\subsubsection{Global stability at DFE}

To present GAS at DFE point, we reproduce the model (1.1) in the following equations utilizing the method described by Castillo-Chavez et al. in his work.

1. $\frac{d Y}{d t}=K(Y, Z)$,

2. $\frac{d Z}{d t}=M(Y, Z), M(Y, 0)=0$,

where $Y \in \mathbb{R}$ and $Y=S$ represents the number of uninfected individuals and $Z \in \mathbb{R}^{5}$, where $Z=(E, I, A, Q, H)$ represents the number of exposed, symptomatic, asymptomatic, 
Quarantine, and hospitalized individuals. The final equation of model (1.1) is omitted since it is unconnected to the others.

$$
P_{o}=\left(Y_{o}, 0\right)=\left(\frac{\lambda}{\tau}, 0\right) .
$$

Under the following two conditions, we present the GAS at DFE.

1. $\frac{d Y}{d t}=K(Y, Z)=0 ; Y_{o}$ is globally asymptotically stable.

2. $\frac{d Z}{d t}=G(Y, Z)=B Z-\bar{G}(Y, Z)$ where $\bar{G}(Y, 0) \geq 0$ for $(Y, \tau) \in \mho$.

where $B=D_{E} G\left(Y_{o}, 0\right)$ is a M-matrix and $\mho=\left\{(S, E, I, A, Q, H) \in \mathbb{R}_{+}^{6} 0 \leq S+E+\right.$ $\left.I+A+Q+H \leq \frac{\lambda}{\tau}\right\}$ is a feasible region.

Theorem 5 If $R_{o}<1$, then the DFE point of system (1.1) represented by $P_{o}=\left(Y_{o}, 0\right)$ will be GAS if conditions (1) and (2) are satisfied.

Proof Let $\mathrm{Y}=\mathrm{S}$ denotes uninfected individuals, $\mathrm{Z}=(\mathrm{E}, \mathrm{I}, \mathrm{A}, \mathrm{Q}, \mathrm{H})$ denotes exposed, symptomatic, asymptomatic, quarantine, and hospitalized people and $P_{o}=\left(Y_{o}, 0\right)$ is the DFE, then

$$
\frac{d Y}{d t}=\frac{d S}{d t}=K(Y, Z)=\lambda-\left(\alpha_{1} I+\alpha_{2} A+\alpha_{3} H\right) S-\tau S,
$$

if $S=S_{o}$ and $K(Y, 0)=0$, then

$$
\frac{d Y}{d t}=K(Y, 0)=0=\lambda-\tau S_{o}(t) .
$$

As $t \rightarrow \infty$, and $Y \rightarrow Y_{o}$. Therefore, $Y=Y_{o}=S_{o}$ is globally asymptotically stable. Here condition 1 is satisfied.

Consider

$$
\frac{\mathrm{d} Z}{\mathrm{~d} t}=B Z-\bar{G}(Y, \tau),
$$

where

$$
\begin{aligned}
B & =\left(\begin{array}{ccccc}
-L_{1} & \alpha_{1} S_{o} & \alpha_{2} S_{o} & 0 & \alpha_{3} S_{o} \\
\kappa_{1} & -L_{2} & \sigma & 0 & 0 \\
\kappa_{2} & 0 & -L_{3} & 0 & 0 \\
0 & \beta_{2} & \gamma_{1} & -L_{4} & 0 \\
0 & \beta_{1} & 0 & v_{1} & -L_{5}
\end{array}\right) \\
Z & =\left(\begin{array}{c}
E \\
I \\
A \\
Q \\
H
\end{array}\right)
\end{aligned}
$$

and

$$
\bar{G}(Y, Z)=\left(\begin{array}{c}
\left(\alpha_{1} I+\alpha_{2} A+\alpha_{3} H\right)\left(S_{o}-S\right) \\
0 \\
0 \\
0 \\
0
\end{array}\right)
$$


So,

$$
S+E+I+A+Q+H \leq \frac{\lambda}{\tau} ; \bar{G}(Y, \tau) \geq 0
$$

. Hence B is M-matrix, Conditions 1 and 2 are satisfied. Thus, $P_{o}$ is GAS.

3.7 Global stability at EE

For GAS of the model (1.1) at EE point $P^{*}$, we prove the following theorem.

Theorem 6 If $R_{O}>1$, then EE point $P^{*}$ of the model (1.1) is GAS stable and unstable if $R_{o}<1$.

Proof Take a Volterra-type Lyapunov function as

$$
\begin{aligned}
F(S, E, I, A, Q, R)= & \left(S-S^{*}-S^{*} \log \frac{S}{S^{*}}\right)+\left(E-E^{*}-E^{*} \log \frac{E}{E^{*}}\right) \\
& +\left(I-I^{*}-I^{*} \log \frac{I}{I^{*}}\right)+\left(A-A^{*}-A^{*} \log \frac{A}{A^{*}}\right) \\
& +\left(Q-Q^{*}-Q^{*} \log \frac{Q}{Q^{*}}\right)+\left(R-R^{*}-R^{*} \log \frac{R}{R^{*}}\right),
\end{aligned}
$$

$\forall(S, E, I, A, Q, H, R) \in \mathbb{R}^{7}$.

$$
\begin{aligned}
\frac{d F}{d t}= & \left(\frac{S-S^{*}}{S}\right) S^{\prime}+\left(\frac{E-E^{*}}{E}\right) E^{\prime}+\left(\frac{I-I^{*}}{I}\right) I^{\prime}+\left(\frac{A-A^{*}}{A}\right) A^{\prime}+\left(\frac{Q-Q^{*}}{Q}\right) Q^{\prime} \\
& +\left(\frac{H-H^{*}}{H}\right) H^{\prime}+\left(\frac{R-R^{*}}{R}\right) R^{\prime},
\end{aligned}
$$

Substitute $S^{\prime}, E^{\prime}, I^{\prime}, A^{\prime}, Q^{\prime}, H^{\prime}, R^{\prime}$ and after re-arrange $\frac{d F}{d t}$, we get

$$
\begin{aligned}
\frac{d F}{d t}= & \left(\frac{S-S^{*}}{S}\right)\left(\lambda-\left(S-S^{*}\right)\left(\alpha_{1} I+\alpha_{2} A+\alpha_{3} H\right)-\tau\left(S-S^{*}\right)\right. \\
& \left.-S^{*}\left(\alpha_{1} I+\alpha_{2} A+\alpha_{3} H+\tau\right)\right) \\
& +\left(\frac{E-E^{*}}{E}\right)\left(S\left(\alpha_{1} I+\alpha_{2} A+\alpha_{3} H\right)-\left(E-E^{*}\right)\left(\kappa_{1}+\kappa_{2}+\tau\right)\right. \\
& \left.-E^{*}\left(\kappa_{1}+\kappa_{2}+\tau\right)\right) \\
& +\left(\frac{I-I^{*}}{I}\right)\left(\left(\kappa_{1} E+\sigma A-\left(I-I^{*}\right)\left(\beta_{1}+\beta_{2}+\beta_{3}+\delta_{I}+\tau\right)\right.\right. \\
& \left.-I^{*}\left(\beta_{1}+\beta_{2}+\beta_{3}+\delta_{I}+\tau\right)\right) \\
& +\left(\frac{A-A^{*}}{A}\right)\left(\kappa_{2} E-\left(A-A^{*}\right)\left(\gamma_{1}+\gamma_{2}+\sigma+\tau\right)-A^{*}\left(\gamma_{1}+\gamma_{2}+\sigma+\tau\right)\right) \\
& +\left(\frac{Q-Q^{*}}{Q}\right)\left(\beta_{2} I+\gamma_{1} A-\left(Q-Q^{*}\right)\left(\nu_{1}+\nu_{2}+\delta_{Q}+\tau\right)\right. \\
& \left.-Q^{*}\left(v_{1}+v_{2}+\delta_{Q}+\tau\right)\right)
\end{aligned}
$$




$$
\begin{aligned}
& +\left(\frac{H-H^{*}}{H}\right)\left(\beta_{1} I+v_{1} Q-\left(H-H^{*}\right)\left(\vartheta+\delta_{H}+\tau\right)-H^{*}\left(\vartheta+\delta_{H}+\tau\right)\right) \\
& +\left(\frac{R-R^{*}}{R}\right)\left(\beta_{3} I+\gamma_{2} A+v_{2} Q+\vartheta H-\tau\left(R-R^{*}\right)-\tau R^{*}\right) .
\end{aligned}
$$

We can write the above equation as:

$$
\frac{d F}{d t}=\delta_{1}-\delta_{2}
$$

where

$$
\begin{aligned}
\delta_{1}= & \lambda+\frac{\left(S^{*}\right)^{2}}{S}\left(\alpha_{1} I+\alpha_{2} A+\alpha_{3} H+\tau\right)+S\left(\alpha_{1} I+\alpha_{2} A+\alpha_{3} H\right) \\
& +\frac{\left(E^{*}\right)^{2}}{E}\left(\kappa_{1}+\kappa_{2}+\tau\right)+\kappa_{1} E+\sigma A+\frac{\left(I^{*}\right)^{2}}{I}\left(\beta_{1}+\beta_{2}+\beta_{3}+\delta_{I}+\tau\right)+\kappa_{2} E \\
& +\frac{\left(A^{*}\right)^{2}}{A}\left(\gamma_{1}+\gamma_{2}+\sigma+\tau\right)+\beta_{2} I+\gamma_{1} A+\frac{\left(Q^{*}\right)^{2}}{Q}\left(v_{1}+\nu_{2}+\delta_{Q}+\tau\right) \\
& +\beta_{1} I+v_{1} Q+\frac{\left(H^{*}\right)^{2}}{H}\left(\vartheta+\delta_{H}+\tau\right)+\beta_{3} I+\gamma_{2} A+v_{2} Q+\vartheta H+\frac{\left(R^{*}\right)^{2}}{R} \tau,
\end{aligned}
$$

and

$$
\begin{aligned}
\delta_{2}= & \frac{\left(S-S^{*}\right)^{2}}{S}\left(\alpha_{1} I+\alpha_{2} A+\alpha_{3} H+\tau\right)+\left(\frac{\lambda S^{*}}{S}+S^{*}\left(\alpha_{1} I+\alpha_{2} A+\alpha_{3} H+\tau\right)\right) \\
& +\frac{\left(E-E^{*}\right)^{2}}{E}\left(\kappa_{1}+\kappa_{2}+\tau\right)+\left(\frac{E^{*}}{E} S\left(\alpha_{1} I+\alpha_{2} A+\alpha_{3} H\right)+E^{*}\left(\kappa_{1}+\kappa_{2}+\tau\right)\right) \\
& +\frac{\left(I-I^{*}\right)^{2}}{I}\left(\beta_{1}+\beta_{2}+\beta_{3}+\delta_{I}+\tau\right) \\
& +\left(\frac{I^{*}}{I}\left(\kappa_{1} E+\sigma A\right)+I^{*}\left(\beta_{1}+\beta_{2}+\beta_{3}+\delta_{I}+\tau\right)\right) \\
& +\frac{\left(A-A^{*}\right)^{2}}{A}\left(\gamma_{1}+\gamma_{2}+\sigma+\tau\right)+\left(\frac{A^{*}}{A} \kappa_{2} E+A^{*}\left(\gamma_{1}+\gamma_{2}+\sigma+\tau\right)\right) \\
& +\frac{\left(Q-Q^{*}\right)^{2}}{Q}\left(v_{1}+v_{2}+\delta_{Q}+\tau\right)+\left(\frac{Q^{*}}{Q}\left(\beta_{2} I+\gamma_{1} A\right)+Q^{*}\left(v_{1}+v_{2}+\delta_{Q}+\tau\right)\right) \\
& +\frac{\left(H-H^{*}\right)^{2}}{H}\left(\vartheta+\delta_{H}+\tau\right)+\left(\frac{H^{*}}{H}\left(\beta_{1} I+v_{1} Q\right)+H^{*}\left(\vartheta+\delta_{H}+\tau\right)\right) \\
& +\frac{\left(R-R^{*}\right)^{2}}{H} \tau+\left(\frac{R^{*}}{R}\left(\beta_{3} I+\gamma_{2} A+v_{2} Q+\vartheta H\right)+R^{*} \tau\right) .
\end{aligned}
$$

All the parameters are positive, thus, $\frac{d F}{d t}<0$, when $\delta_{1}<\delta_{2}, \frac{d F}{d t}=0$ iff $S=S^{*}, E=$ $E^{*}, I=I^{*}, A=A^{*}, Q=Q^{*}, H=H^{*}, R=R^{*}$. Hence, by Lasalle's invariance Principle, $\mathrm{EE}$ is GAS. 


\section{Analysis of fuzzy fractional model (3)}

In this part of paper, we give the existence and uniqueness of solutions to the model (3) and we have given a method for finding a semi-analytic solution of model by (3) by using the fuzzy hybrid Laplace transform. (Table 1).

\subsection{Existence and uniqueness of fuzzy fractional model}

Fixed point theory plays a important role in order to elaborate the existence and uniqueness of the model (3). Let us write the model (3) as

$$
\left\{\begin{array}{l}
\varphi(t, S(t))=\tilde{\lambda}-\left(\tilde{\alpha_{1}} I+\tilde{\alpha_{2}} A+\tilde{\alpha_{2}} H\right) S-\tilde{\tau} S, \\
\phi(t, E(t))=\left(\tilde{\alpha_{1}} I+\tilde{\alpha_{2}} A+\tilde{\alpha_{2}} H\right) S-\left(\tilde{\kappa_{1}}+\tilde{\kappa_{2}}+\tilde{\tau}\right) E, \\
\xi(t, I(t))=\tilde{\kappa_{1}} E+\tilde{\sigma} A-\left(\tilde{\beta_{1}}+\tilde{\beta_{2}}+\tilde{\beta_{3}}+\tilde{\delta}_{I}+\tilde{\tau}\right) I, \\
\eta(t, A(t))=\tilde{\kappa_{2}} E-\left(\tilde{\gamma_{1}}+\tilde{\gamma_{2}}+\tilde{\sigma}+\tilde{\tau}\right) A, \\
\rho(t, Q(t))=\tilde{\beta_{2}} I+\tilde{\gamma_{1}} A-\left(\tilde{\nu_{1}}+\tilde{\nu_{2}}+\tilde{\delta} Q+\tilde{\tau}\right) Q, \\
\gamma(t, H(t))=\tilde{\beta_{1}} I+\tilde{\nu_{1}} Q-\left(\tilde{\vartheta}+\tilde{\delta}_{H}+\tilde{\tau}\right) H, \\
\sigma(t, R(t))=\tilde{\beta_{3}} I+\tilde{\gamma_{2}} A+\tilde{\gamma_{2}} Q+\tilde{\vartheta} H-\tilde{\tau} R,
\end{array}\right.
$$

where $\varphi, \phi, \xi, \eta, \rho, \gamma, \sigma$ are the fuzzy functions. The model (3) gets the form

$$
\begin{cases}D_{t}^{y} S(t) & =\varphi(t, S(t)) \\ D_{t}^{y} E(t) & =\phi(t, E(t)) \\ D_{t}^{y} I(t) & =\xi(t, I(t)) \\ D_{t}^{y} A(t) & =\eta(t, A(t)) \\ D_{t}^{y} Q(t) & =\rho(t, Q(t)) \\ D_{t}^{y} H(t) & =\gamma(t, H(t)) \\ D_{t}^{y} R(t) & =\sigma(t, R(t))\end{cases}
$$

subject to the fuzzy initial conditions

$$
\begin{aligned}
\tilde{S}(0, \zeta) & =(\underline{S}(0, \zeta), \bar{S}(0, \zeta)), \\
\tilde{E}(0, \zeta) & =(\underline{E}(0, \zeta), \bar{E}(0, \zeta)), \\
\tilde{I}(0, \zeta) & =(\underline{I}(0, \zeta), \bar{I}(0, \zeta)), \\
\tilde{A}(0, \zeta) & =(\underline{A}(0, \zeta), \bar{A}(0, \zeta)), \\
\tilde{Q}(0, \zeta) & =(\underline{Q}(0, \zeta), \bar{Q}(0, \zeta)), \\
\tilde{H}(0, \zeta) & =(\underline{H}(0, \zeta), \bar{H}(0, \zeta)), \\
\tilde{R}(0, \zeta) & =(\underline{R}(0, \zeta), \bar{R}(0, \zeta)) .
\end{aligned}
$$


Now, implementing the FFI $I^{y}$, we achieve

$$
\left\{\begin{array}{l}
S(t)=\tilde{S}(0, \zeta)+\frac{1}{\Gamma(y)} \int_{0}^{t}(t-s)^{y-1} \varphi(s, S(s)) d s \\
E(t)=\tilde{E}(0, \zeta)+\frac{1}{\Gamma(y)} \int_{0}^{t}(t-s)^{y-1} \phi(s, E(s)) d s \\
I(t)=\tilde{I}(0, \zeta)+\frac{1}{\Gamma(y)} \int_{0}^{t}(t-s)^{y-1} \xi(s, I(s)) d s \\
A(t)=\tilde{A}(0, \zeta)+\frac{1}{\Gamma(y)} \int_{0}^{t}(t-s)^{y-1} \eta(s, A(s)) d s \\
Q(t)=\tilde{Q}(0, \zeta)+\frac{1}{\Gamma(y)} \int_{0}^{t}(t-s)^{y-1} \rho(s, Q(s)) d s \\
H(t)=\tilde{H}(0, \zeta)+\frac{1}{\Gamma(y)} \int_{0}^{t}(t-s)^{y-1} \gamma(s, H(s)) d s \\
R(t)=\tilde{R}(0, \zeta)+\frac{1}{\Gamma(y)} \int_{0}^{t}(t-s)^{y-1} \sigma(s, R(s)) d s
\end{array}\right.
$$

Define a Banach space as $B=B_{1} \times B_{2} \times B_{3} \times B_{4} \times B_{5} \times B_{6} \times B_{7}$, we have under the fuzzy norm:

$$
\begin{aligned}
& \|S(t), E(t), I(t), A(t), Q(t), H(t), R(t)\| \\
& \quad=\max _{t \in[0, T]}[S(t)+E(t)+I(t)+A(t)+Q(t)+H(t)+R(t)]
\end{aligned}
$$

Let us define a mapping from $B$ to $B$ as:

$$
\tilde{\hbar}(t)=\tilde{\hbar}(0, \zeta)+\frac{1}{\Gamma y} \int_{0}^{t}(t-\wp)^{y-1} \ominus(\wp, \tilde{\hbar}(\wp)) d \wp
$$

where

$$
\begin{gathered}
\tilde{\hbar(t)}=\left(\begin{array}{l}
S(t) \\
E(t) \\
I(t) \\
A(t) \\
Q(t) \\
H(t) \\
R(t)
\end{array}\right), \\
\tilde{\hbar}(0, \zeta)=\left(\begin{array}{l}
S(0, \zeta) \\
E(0, \zeta) \\
I(0, \zeta) \\
A(0, \zeta) \\
Q(0, \zeta) \\
H(0, \zeta) \\
R(0, \zeta)
\end{array}\right),
\end{gathered}
$$


and

$$
\ominus(t, \tilde{\hbar}(t))=\left(\begin{array}{c}
\varphi(t, S(t)) \\
\phi(t, E(t)) \\
\xi(t, I(t)) \\
\eta(t, A(t)) \\
\rho(t, Q(t)) \\
\gamma(t, H(t)) \\
\sigma(t, R(t))
\end{array}\right) .
$$

Let us impose some conditions on the nonlinear function $\ominus$ as follows: There exist constant $K_{\hbar}>0$ such that for each $\tilde{\hbar_{1}}(t), \tilde{\hbar_{2}}(t) \in B$, we have

$$
\left|\ominus\left(t, \tilde{\hbar_{1}}(t)\right)-\Theta\left(t, \tilde{\hbar_{2}}(t)\right)\right| \leq K_{\hbar}\left|\tilde{\hbar_{1}}(t)-\tilde{\hbar_{2}}(t)\right| .
$$

There exist a constant $M_{\hbar}>0$ and $N_{\hbar}>0$ such that:

$$
\left.|\ominus(t, \tilde{\hbar(}(t))| \leq M_{\hbar} \mid \tilde{\hbar(}(t)\right) \mid+N_{\hbar} .
$$

Theorem 7 Assume that (27) holds. Then, considered fuzzy fractional model has at least one solutions.

Proof Let $\mathcal{S}=\{\tilde{\hbar}(t) \in B: \| \tilde{\hbar}(t)) \| \leq r\} \subset \mathbb{B}$ be a closed and convex fuzzy set, and Define a mapping $\varphi: \mathcal{S} \rightarrow \mathcal{S}$ such that

$$
\varphi(\tilde{\hbar}(t))=\tilde{\hbar}(0, \zeta)+\frac{1}{\Gamma y} \int_{0}^{t}(t-\wp)^{y-1} \ominus(\wp, \tilde{\hbar}(\wp)) d \wp .
$$

For any $\tilde{\hbar}(t) \in \mathcal{S}$, we have

$$
\begin{aligned}
\|\varphi(\tilde{\hbar}(t))\|= & \max _{t \in[0, T]}\left|\tilde{\hbar}(0, \zeta)+\frac{1}{\Gamma y} \int_{0}^{t}(t-\wp)^{y-1} \ominus(\wp, \tilde{\hbar}(\wp)) d \wp\right| \\
& \leq|\tilde{\hbar}(0, \zeta)|+\frac{1}{\Gamma y} \int_{0}^{t}(t-\wp)^{y-1}|\ominus(\wp, \tilde{\hbar}(\wp))| d \wp \\
& \leq|\tilde{\hbar}(0, \zeta)|+\frac{1}{\Gamma y} \int_{0}^{t}(t-\wp)^{y-1}\left[M_{\hbar}|\tilde{\hbar}(t)|+N_{\hbar}\right] d \wp \\
& \leq|\tilde{\hbar}(0, \zeta)|+\frac{t^{y}}{\Gamma(y+1)}\left[M_{\hbar}|\tilde{\hbar}(t)|+N_{\hbar}\right] .
\end{aligned}
$$


If we look back at the last equation with $\varphi(\mathcal{S}) \subset \mathcal{S}$, we come to a result that operator $\varphi$ is bounded. Now, we show that the operator $\varphi$ is completely continuous. Let $\varphi_{1}, \varphi_{2} \in[0, T]$ be such that $\varphi_{1}<\varphi_{2}$, then

$$
\begin{aligned}
& \left\|\varphi(\tilde{\hbar}(t))\left(\varphi_{2}\right)-\varphi(\tilde{\hbar}(t))\left(\varphi_{1}\right)\right\|=\mid \frac{1}{\Gamma y} \int_{0}^{\varphi}\left(\varphi_{2}-\wp\right)^{y-1} \ominus(\wp, \tilde{\hbar}(\wp)) d \wp \\
& \quad-\frac{1}{\Gamma y} \int_{0}^{\varphi}\left(\varphi_{1}-\wp\right)^{y-1} \ominus(\wp, \tilde{\hbar}(\wp)) d \wp \mid \\
& \leq\left[\varphi_{2}^{y}-\varphi_{1}^{y}\right] \frac{\left.\left[M_{\hbar} \mid \tilde{\hbar}(\wp)\right) \mid+N_{\hbar}\right]}{\Gamma(y+1)},
\end{aligned}
$$

while in the recent discussion from the last expression, we see that

$$
\left\|\varphi(\tilde{\hbar}(t))\left(\varphi_{2}\right)-\varphi(\tilde{\hbar}(t))\left(\varphi_{1}\right)\right\| \rightarrow 0,
$$

as $\varphi_{2} \rightarrow \varphi_{1}$. Hence, the operator $\varphi$ is equi-continuous. By Arzela-Ascoli theorem, the operator $\varphi$ is completely continuous. By Schauder's fixed point theorem, the considered fuzzy fractional model possesses at least one solution.

Theorem 8 The considered fuzzy fractional model has a unique solution if (26) holds and

$$
\tau^{y} \mathcal{Q}_{\aleph}<\Gamma(y+1) .
$$

Proof Let $\tilde{\hbar_{1}}(t), \tilde{\hbar_{2}}(t) \in B$, then

$$
\begin{aligned}
& \left\|\varphi\left(\hbar_{i_{1}}(t)\right)-\varphi\left(\hbar_{i_{2}}(t)\right)\right\|=\max _{t \in[0, T]} \mid \frac{1}{\Gamma y} \int_{0}^{t}(t-\wp)^{y-1} \ominus(\wp, \tilde{\hbar}(\wp)) d \wp \\
& \quad-\frac{1}{\Gamma y} \int_{0}^{t}(t-\wp)^{y-1} \ominus(\wp, \tilde{\hbar}(\wp)) d \wp \mid \\
& \leq \frac{t^{y}}{\Gamma(y+1)} \mathcal{Q}_{\aleph}\left|\tilde{\hbar}_{1}(t)-\tilde{\hbar_{2}}(t)\right| .
\end{aligned}
$$

Hence, $\varphi$ satisfies the contraction condition. Hence, by Banach fixed result, the considered fuzzy model has a unique solutions.

\section{Scheme for the solution}

The main target of this section is to find the solution procedure for the considered fuzzy fractional model by Laplace transform (LT). Implementing the fuzzy LT, one can get

$$
\begin{aligned}
\mathcal{L}\left[D_{z}^{y} S(t)\right] & =\mathcal{L}[\varphi(t, S(t)], \\
\mathcal{L}\left[D_{z}^{y} E(t)\right] & =\mathcal{L}[\phi(t, E(t)], \\
\mathcal{L}\left[D_{z}^{y} I(t)\right] & =\mathcal{L}[\xi(t, I(t)], \\
\mathcal{L}\left[D_{z}^{y} A(t)\right] & =\mathcal{L}[\eta(t, A(t)], \\
\mathcal{L}\left[D_{z}^{y} Q(t)\right] & =\mathcal{L}[\rho(t, Q(t)],
\end{aligned}
$$


Table 1 Parameter values used for simulations

\begin{tabular}{lll}
\hline Notation & Parameters description & Numerical value \\
\hline$\lambda$ & Recruitment rate into susceptible population & $\tau * N(0)$ \\
$S^{o}$ & Initial stage of Susceptible individuals & 0.4 \\
$E^{o}$ & Initial stage of Exposed individuals & 0.2 \\
$I^{o}$ & Initial stage of symptomatic individuals & 0.1 \\
$A^{o}$ & Initial stage of asymptomatic individuals & 0.1 \\
$Q^{o}$ & Initial stage of Quarantine individuals & 0.1 \\
$H^{o}$ & Initial stage of hospitalized individuals & 0.05 \\
$R^{o}$ & Initial stage of Recovered individuals & 0.05 \\
$\alpha_{1}$ & Effective contact rate from symptomatic to susceptible & 0.022 \\
$\alpha_{2}$ & Effective contact rate from asymptomatic to susceptible & 0.1622 \\
$\alpha_{3}$ & Effective contact rate from hospitalized to susceptible & 0.0131 \\
$\tau$ & Natural death rate & 1 \\
$\kappa_{1}$ & Rate of becoming symptomatic upon infection & $365 \times 67.7$ \\
$\kappa_{2}$ & Rate of becoming asymptomatic upon infection & 0.2350 \\
$\sigma$ & Rate of asymptomatic becomes symptomatic & 0.0 .2691 \\
$\beta_{1}$ & Rate of symptomatic becomes hospitalized & 0.0632 \\
$\beta_{2}$ & Rate of symptomatic becomes quarantined & 0.4507 \\
$\beta_{3}$ & Rate of symptomatic of becoming recovered & 0.3716 \\
$\gamma_{1}$ & Rate of asymptomatic becomes quarantined & 0.6381 \\
$\gamma_{2}$ & Rate of asymptomatic of becoming recovered & 0.2310 \\
$\nu_{1}$ & Rate of quarantine people becoming hospitalized & 0.0803 \\
$\nu_{2}$ & Rate of quarantine people becoming recovered & 0.1396 \\
$\vartheta$ & Rate of becoming recovered from hospitalized & 0.4870 \\
$\delta_{I}$ & Death rate of symptomatic due to disease & 0.5431 \\
$\delta_{Q}$ & Death rate of quarantine to disease & 0.0002 \\
$\delta_{H}$ & Death rate of hospitalized due to disease & 0.01 \\
\hline & & 0.01 \\
\hline
\end{tabular}

$$
\begin{aligned}
\mathcal{L}\left[D_{z}^{y} H(t)\right] & =\mathcal{L}[\gamma(t, H(t)], \\
\mathcal{L}\left[D_{z}^{y} R(t)\right] & =\mathcal{L}[\sigma(t, R(t)] .
\end{aligned}
$$

Using initial conditions, we acquire

$$
\begin{aligned}
s^{y} \mathcal{L}\left[D_{t}^{y} S(t)\right] & =s^{y-1} \tilde{S}(0, \zeta)+\mathcal{L}[\varphi(t, S(t)], \\
s^{y} \mathcal{L}\left[D_{t}^{y} E(t)\right] & =s^{y-1} \tilde{E}(0, \zeta)+\mathcal{L}[\phi(t, E(t)], \\
s^{y} \mathcal{L}\left[D_{t}^{y} I(t)\right] & =s^{y-1} \tilde{I}(0, \zeta)+\mathcal{L}[\xi(t, I(t)], \\
s^{y} \mathcal{L}\left[D_{t}^{y} A(t)\right] & =s^{y-1} \tilde{A}(0, \zeta)+\mathcal{L}[\eta(t, A(t)], \\
s^{y} \mathcal{L}\left[D_{t}^{y} Q(t)\right] & =s^{y-1} \tilde{Q}(0, \zeta)+\mathcal{L}[\rho(t, Q(t)], \\
s^{y} \mathcal{L}\left[D_{t}^{y} H(t)\right] & =s^{y-1} \tilde{H}(0, \zeta)+\mathcal{L}[\gamma(t, H(t)], \\
s^{y} \mathcal{L}\left[D_{t}^{y} R(t)\right] & =s^{y-1} \tilde{R}(0, \zeta)+\mathcal{L}[\sigma(t, R(t)] .
\end{aligned}
$$


Equivalently, we have

$$
\begin{aligned}
\mathcal{L}\left[D_{t}^{y} S(t)\right] & =\frac{1}{s} \tilde{S}(0, \zeta)+\frac{1}{s^{y}} \mathcal{L}[\varphi(t, S(t)], \\
\mathcal{L}\left[D_{t}^{y} E(t)\right] & =\frac{1}{s} \tilde{E}(0, \zeta)+\frac{1}{s^{y}} \mathcal{L}[\phi(t, E(t)], \\
\mathcal{L}\left[D_{t}^{y} I(t)\right] & =\frac{1}{s} \tilde{I}(0, \zeta)+\frac{1}{s^{y}} \mathcal{L}[\xi(t, I(t)], \\
\mathcal{L}\left[D_{t}^{y} A(t)\right] & =\frac{1}{s} \tilde{A}(0, \zeta)+\frac{1}{s^{y}} \mathcal{L}[\eta(t, A(t)], \\
\mathcal{L}\left[D_{t}^{y} Q(t)\right] & =\frac{1}{s} \tilde{Q}(0, \zeta)+\frac{1}{s^{y}} \mathcal{L}[\rho(t, Q(t)], \\
\mathcal{L}\left[D_{t}^{y} H(t)\right] & =\frac{1}{s} \tilde{H}(0, \zeta)+\frac{1}{s^{y}} \mathcal{L}[\gamma(t, H(t)], \\
\mathcal{L}\left[D_{t}^{y} R(t)\right] & =\frac{1}{s} \tilde{R}(0, \zeta)+\frac{1}{s^{y}} \mathcal{L}[\sigma(t, R(t)] .
\end{aligned}
$$

Consider the infinite series solutions as:

$$
\begin{aligned}
& S(t)=\sum_{n=0}^{\infty} S_{n}(t), \quad E(t)=\sum_{n=0}^{\infty} E_{n}(t), \quad I(t)=\sum_{n=0}^{\infty} I_{n}(t), \\
& A(t)=\sum_{n=0}^{\infty} A_{n}(t), \quad Q(t)=\sum_{n=0}^{\infty} Q_{n}(t), \quad H(t)=\sum_{n=0}^{\infty} H_{n}(t), \\
& R(t)=\sum_{n=0}^{\infty} R_{n}(t) .
\end{aligned}
$$

The nonlinear terms in the model are decomposed as:

$$
S(t) I(t)=\sum_{n=0}^{\infty} \mathrm{Ł}_{1, n}, \quad S(t) A(t)=\sum_{n=0}^{\infty} \mathrm{Ł}_{2, n}, \quad S(t) Q(t)=\sum_{n=0}^{\infty} \mathrm{Ł}_{3, n},
$$

where $Ł_{1, n}, Ł_{2, n}, Ł_{3, n}$ are Adomian polynomials, representing nonlinear terms, so the last equations becomes

$$
\begin{aligned}
\mathcal{L}\left[\sum_{n=0}^{\infty} S_{n}(t)\right] & =\frac{1}{s} \tilde{S}(0, \zeta)+\frac{1}{s^{y}} \mathcal{L}\left[\varphi\left(t, \sum_{n=0}^{\infty} S_{n}(t)\right],\right. \\
\mathcal{L}\left[\sum_{n=0}^{\infty} E_{n}(t)\right] & =\frac{1}{s} \tilde{E}(0, \zeta)+\frac{1}{s^{y}} \mathcal{L}\left[\phi\left(t, \sum_{n=0}^{\infty} E_{n}(t)\right],\right. \\
\mathcal{L}\left[\sum_{n=0}^{\infty} I_{n}(t)\right] & =\frac{1}{s} \tilde{I}(0, \zeta)+\frac{1}{s^{y}} \mathcal{L}\left[\xi\left(t, \sum_{n=0}^{\infty} I_{n}(t)\right],\right. \\
\mathcal{L}\left[\sum_{n=0}^{\infty} A_{n}(t)\right] & =\frac{1}{s} \tilde{A}(0, \zeta)+\frac{1}{s^{y}} \mathcal{L}\left[\eta\left(t, \sum_{n=0}^{\infty} A_{n}(t)\right],\right. \\
\mathcal{L}\left[\sum_{n=0}^{\infty} Q_{n}(t)\right] & =\frac{1}{s} \tilde{Q}(0, \zeta)+\frac{1}{s^{y}} \mathcal{L}\left[\rho\left(t, \sum_{n=0}^{\infty} Q_{n}(t)\right],\right.
\end{aligned}
$$




$$
\begin{aligned}
& \mathcal{L}\left[\sum_{n=0}^{\infty} H_{n}(t)\right]=\frac{1}{s} \tilde{H}(0, \zeta)+\frac{1}{s^{y}} \mathcal{L}\left[\gamma\left(t, \sum_{n=0}^{\infty} H_{n}(t)\right],\right. \\
& \mathcal{L}\left[\sum_{n=0}^{\infty} R_{n}(t)\right]=\frac{1}{s} \tilde{R}(0, \zeta)+\frac{1}{s^{y}} \mathcal{L}\left[\sigma\left(t, \sum_{n=0}^{\infty} R_{n}(t)\right] .\right.
\end{aligned}
$$

Taking inverse LT, we have

$$
\begin{aligned}
& \sum_{n=0}^{\infty} S_{n}(t)=\tilde{S}(0, \zeta)+\mathcal{L}^{-1}\left[\frac{1}{s^{y}} \mathcal{L}\left[\varphi\left(t, \sum_{n=0}^{\infty} S_{n}(t)\right]\right]\right. \\
& \sum_{n=0}^{\infty} E_{n}(t)=\tilde{E}(0, \zeta)+\mathcal{L}^{-1}\left[\frac{1}{s^{y}} \mathcal{L}\left[\phi\left(t, \sum_{n=0}^{\infty} E_{n}(t)\right]\right]\right. \\
& \sum_{n=0}^{\infty} I_{n}(t)=\tilde{I}(0, \zeta)+\mathcal{L}^{-1}\left[\frac{1}{s^{y}} \mathcal{L}\left[\xi\left(t, \sum_{n=0}^{\infty} I_{n}(t)\right]\right]\right. \\
& \sum_{n=0}^{\infty} A_{n}(t)=\tilde{A}(0, \zeta)+\mathcal{L}^{-1}\left[\frac{1}{s^{y}} \mathcal{L}\left[\eta\left(t, \sum_{n=0}^{\infty} A_{n}(t)\right]\right]\right. \\
& \sum_{n=0}^{\infty} Q_{n}(t)=\tilde{Q}(0, \zeta)+\mathcal{L}^{-1}\left[\frac{1}{s^{y}} \mathcal{L}\left[\rho\left(t, \sum_{n=0}^{\infty} Q_{n}(t)\right]\right]\right. \\
& \sum_{n=0}^{\infty} H_{n}(t)=\tilde{H}(0, \zeta)+\mathcal{L}^{-1}\left[\frac{1}{s^{y}} \mathcal{L}\left[\gamma\left(t, \sum_{n=0}^{\infty} H_{n}(t)\right]\right]\right. \\
& \sum_{n=0}^{\infty} R_{n}(t)=\tilde{R}(0, \zeta)+\mathcal{L}^{-1}\left[\frac{1}{s^{y}} \mathcal{L}\left[\sigma\left(t, \sum_{n=0}^{\infty} R_{n}(t)\right]\right]\right.
\end{aligned}
$$

Comparing the terms in their parametric form, we have

$$
\begin{aligned}
\underline{S}_{0}(t) & =\underline{S}(0, \zeta), \bar{S}_{0}(t)=\bar{S}(0, \zeta), \\
\underline{E}_{0}(t) & =\underline{E}(0, \zeta), \bar{E}_{0}(t)=\bar{E}(0, \zeta), \\
\underline{I}_{0}(t) & =\underline{I}(0, \zeta), \bar{I}_{0}(t)=\bar{I}(0, \zeta), \\
\underline{A}_{0}(t) & =\underline{A}(0, \zeta), \bar{A}_{0}(t)=\bar{A}(0, \zeta), \\
\underline{Q}_{0}(t) & =\underline{Q}(0, \zeta), \bar{Q}_{0}(t)=\bar{Q}(0, \zeta), \\
\underline{H}_{0}(t) & =\underline{H}(0, \zeta), \bar{H}_{0}(t)=\bar{H}(0, \zeta), \\
\underline{R}_{0}(t) & =\underline{R}(0, \zeta), \bar{R}_{0}(t)=\bar{R}(0, \zeta),
\end{aligned}
$$

and

$$
\begin{aligned}
& \underline{S}_{1}(t)=\mathcal{L}^{-1}\left[\frac{1}{s^{y}} \mathcal{L}\left[\tilde{\lambda}-\left(\tilde{\alpha_{1}} \underline{I}_{0}+\tilde{\alpha}_{2} \underline{A}_{0}+\tilde{\alpha_{3}} \underline{H}_{0}\right) \underline{S}_{0}-\tilde{\tau} \underline{S}_{0}\right]\right], \\
& \bar{S}_{1}(t)=\mathcal{L}^{-1}\left[\frac{1}{s^{y}} \mathcal{L}\left[\tilde{\lambda}-\left(\tilde{\alpha_{1}} \bar{I}_{0}+\tilde{\alpha_{2}} \bar{A}_{0}+\tilde{\alpha_{3}} \bar{H}_{0}\right) \bar{S}_{0}-\tilde{\tau} \bar{S}_{0}\right]\right] .
\end{aligned}
$$

On the same way, we can find the other terms. Hence, the series solutions is expressed as:

$$
\begin{aligned}
& \underline{S}(t)=\underline{S}_{0}(t)+\underline{S}_{1}(t)+\underline{S}_{2}(t)+\ldots, \\
& \bar{S}(t)=\bar{S}_{0}(t)+\bar{S}_{1}(t)+\bar{S}_{2}(t)+\ldots,
\end{aligned}
$$




$$
\begin{aligned}
& \underline{E}(t)=\underline{E}_{0}(t)+\underline{E}_{1}(t)+\underline{E}_{2}(t)+\ldots, \\
& \bar{E}(t)=\bar{E}_{0}(t)+\bar{E}_{1}(t)+\bar{E}_{2}(t)+\ldots, \\
& \underline{I}(t)=\underline{I}_{0}(t)+\underline{I}_{1}(t)+\underline{I}_{2}(t)+\ldots, \\
& \bar{I}(t)=\bar{I}_{0}(t)+\bar{I}_{1}(t)+\bar{I}_{2}(t)+\ldots, \\
& \underline{A}(t)=\underline{A}_{0}(t)+\underline{A}_{1}(t)+\underline{A}_{2}(t)+\ldots, \\
& \bar{A}(t)=\bar{A}_{0}(t)+\bar{A}_{1}(t)+\bar{A}_{2}(t)+\ldots, \\
& \underline{Q}(t)=\underline{Q}_{0}(t)+\underline{Q}_{1}(t)+\underline{Q}_{2}(t)+\ldots, \\
& \bar{Q}(t)=\bar{Q}_{0}(t)+\bar{Q}_{1}(t)+\bar{Q}_{2}(t)+\ldots, \\
& \underline{H}(t)=\underline{H}_{0}(t)+\underline{H}_{1}(t)+\underline{H}_{2}(t)+\ldots \text {, } \\
& \bar{H}(t)=\bar{H}_{0}(t)+\bar{H}_{1}(t)+\bar{H}_{2}(t)+\ldots, \\
& \underline{R}(t)=\underline{R}_{0}(t)+\underline{R}_{1}(t)+\underline{R}_{2}(t)+\ldots, \\
& \bar{R}(t)=\bar{R}_{0}(t)+\bar{R}_{1}(t)+\bar{R}_{2}(t)+\ldots
\end{aligned}
$$

\section{Numerical results and discussion}

In this part, we compute the terms of the series solution for a specific triangular fuzzy number. Consider the proposed model with initial conditions

$$
\begin{aligned}
& \tilde{S}(0, \zeta)=(\zeta-1,1-\zeta), \tilde{E}(0, \zeta)=(\zeta-1,1-\zeta), \tilde{I}(0, \zeta)=(\zeta-1,1-\zeta), \\
& \tilde{A}(0, \zeta)=(\zeta-1,1-\zeta), \tilde{Q}(0, \zeta)=(\zeta-1,1-\zeta), \tilde{H}(0, \zeta)=(\zeta-1,1-\zeta), \\
& \tilde{R}(0, \zeta)=(\zeta-1,1-\zeta) .
\end{aligned}
$$

Using the above developed procedure and using the ICs, one have

$$
\begin{aligned}
\underline{S}_{0}(t, \zeta) & =(\zeta-1), \quad \bar{S}_{0}(t, \zeta)=(1-\zeta), \\
\underline{E}_{0}(t, \zeta) & =(\zeta-1), \quad \bar{E}_{0}(t, \zeta)=(1-\zeta), \\
\underline{I}_{0}(t, \zeta) & =(\zeta-1), \quad \bar{I}_{0}(t, \zeta)=(1-\zeta), \\
\underline{A}_{0}(t, \zeta) & =(\zeta-1), \quad \bar{A}_{0}(t, \zeta)=(1-\zeta), \\
\underline{Q}_{0}(t, \zeta) & =(\zeta-1), \quad \bar{Q}_{0}(t, \zeta)=(1-\zeta), \\
\underline{H}_{0}(t, \zeta) & =(\zeta-1), \quad \bar{H}_{0}(t, \zeta)=(1-\zeta), \\
\underline{R}_{0}(t, \zeta) & =(\zeta-1), \quad \bar{R}_{0}(t, \zeta)=(1-\zeta) .
\end{aligned}
$$

The second term is given by

$$
\begin{aligned}
& \underline{S}_{1}(t, \zeta)=\left[\tilde{\lambda}-\left[\tilde{\alpha_{1}}+\tilde{\alpha_{2}}+\tilde{\alpha_{3}}\right](\zeta-1)^{2}-\tilde{\tau}(\zeta-1)\right] \frac{t^{y}}{\Gamma(y+1)}, \\
& \bar{S}_{1}(t, \zeta)=\left[\tilde{\lambda}-\left[\tilde{\alpha_{1}}+\tilde{\alpha_{2}}+\tilde{\alpha_{3}}\right](1-\zeta)^{2}-\tilde{\tau}(1-\zeta)\right] \frac{t^{y}}{\Gamma(y+1)}, \\
& \underline{E}_{1}(t, \zeta)=\left[\left[\tilde{\alpha_{1}}+\tilde{\alpha_{2}}+\tilde{\alpha_{3}}\right](\zeta-1)^{2}-\left[\tilde{\kappa_{1}}+\tilde{\kappa_{2}}+\tilde{\tau}\right](\zeta-1)\right] \frac{t^{y}}{\Gamma(y+1)}, \\
& \bar{E}_{1}(t, \zeta)=\left[\left[\tilde{\alpha_{1}}+\tilde{\alpha_{2}}+\tilde{\alpha_{3}}\right](1-\zeta)^{2}-\left[\tilde{\kappa_{1}}+\tilde{\kappa_{2}}+\tilde{\tau}\right](1-\zeta)\right] \frac{t^{y}}{\Gamma(y+1)}, \\
& \underline{I}_{1}(t, \zeta)=\left[\left[\tilde{\kappa_{1}}+\tilde{\sigma}\right](\zeta-1)-\left[\tilde{\beta_{1}}+\tilde{\beta_{2}}+\tilde{\beta_{3}}+\tilde{\delta}_{I}+\tilde{\tau}\right](\zeta-1)\right] \frac{t^{y}}{\Gamma(y+1)},
\end{aligned}
$$




$$
\begin{aligned}
& \bar{I}_{1}(t, \zeta)=\left[\left[\tilde{\kappa_{1}}+\tilde{\sigma}\right](1-\zeta)-\left[\tilde{\beta}_{1}+\tilde{\beta}_{2}+\tilde{\beta}_{3}+\tilde{\delta}_{I}+\tilde{\tau}\right](1-\zeta)\right] \frac{t^{y}}{\Gamma(y+1)}, \\
& \underline{A}_{1}(t, \zeta)=\left[\tilde{\kappa_{2}}(\zeta-1)-\left[\tilde{\gamma_{1}}+\tilde{\gamma_{2}}+\tilde{\sigma}+\tilde{\tau}\right](\zeta-1)\right] \frac{t^{y}}{\Gamma(y+1)}, \\
& \bar{A}_{1}(t, \zeta)=\left[\tilde{\kappa_{2}}(1-\zeta)-\left[\tilde{\gamma_{1}}+\tilde{\gamma_{2}}+\tilde{\sigma}+\tilde{\tau}\right](1-\zeta)\right] \frac{t^{y}}{\Gamma(y+1)}, \\
& \underline{Q}_{1}(t, \zeta)=\left[\left[\tilde{\beta}_{2}+\tilde{\gamma_{1}}\right](\zeta-1)-\left[\tilde{v}_{1}+\tilde{v_{2}}+\tilde{\delta}_{Q}+\tilde{\tau}\right](\zeta-1)\right] \frac{t^{y}}{\Gamma(y+1)}, \\
& \bar{Q}_{1}(t, \zeta)=\left[\left[\tilde{\beta}_{2}+\tilde{\gamma_{1}}\right](1-\zeta)-\left[{\tilde{v_{1}}}+{\tilde{v_{2}}}+\tilde{\delta}_{Q}+\tilde{\tau}\right](1-\zeta)\right] \frac{t^{y}}{\Gamma(y+1)}, \\
& \underline{H}_{1}(t, \zeta)=\left[\left[\tilde{\beta}_{1}+\tilde{v}_{1}\right](\zeta-1)-\left[\tilde{\vartheta}+\tilde{\delta}_{H}+\tilde{\tau}\right](\zeta-1)\right] \frac{t^{y}}{\Gamma(y+1)}, \\
& \bar{H}_{1}(t, \zeta)=\left[\left[\tilde{\beta}_{1}+\tilde{v}_{1}\right](1-\zeta)-\left[\tilde{\vartheta}+\tilde{\delta}_{H}+\tilde{\tau}\right](1-\zeta)\right] \frac{t^{y}}{\Gamma(y+1)}, \\
& \underline{R}_{1}(t, \zeta)=\left[\left[\tilde{\beta_{3}}+\tilde{\gamma_{2}}+\tilde{\nu_{2}}+\tilde{\vartheta}\right](\zeta-1)-\tilde{\tau}(\zeta-1)\right] \frac{t^{y}}{\Gamma(y+1)}, \\
& \bar{R}_{1}(t, \zeta)=\left[\left[\tilde{\beta_{3}}+\tilde{\gamma_{2}}+\tilde{\nu_{2}}+\tilde{\vartheta}\right](1-\zeta)-\tilde{\tau}(1-\zeta)\right] \frac{t^{y}}{\Gamma(y+1)} .
\end{aligned}
$$

For the sake of simplicity, let

$$
\begin{aligned}
& \underline{D_{1}}=\left[\tilde{\lambda}-\left[\tilde{\alpha_{1}}+\tilde{\alpha_{2}}+\tilde{\alpha_{3}}\right](\zeta-1)^{2}-\tilde{\tau}(\zeta-1)\right], \\
& \overline{D_{1}}=\left[\tilde{\lambda}-\left[\tilde{\alpha_{1}}+\tilde{\alpha_{2}}+\tilde{\alpha_{3}}\right](1-\zeta)^{2}-\tilde{\tau}(1-\zeta)\right] \text {, } \\
& \underline{D_{2}}=\left[\left[\tilde{\alpha_{1}}+\tilde{\alpha_{2}}+\tilde{\alpha_{3}}\right](\zeta-1)^{2}-\left[\tilde{\kappa_{1}}+\tilde{\kappa_{2}}+\tilde{\tau}\right](\zeta-1)\right] \text {, } \\
& \overline{D_{2}}=\left[\left[\tilde{\alpha_{1}}+\tilde{\alpha_{2}}+\tilde{\alpha_{3}}\right](1-\zeta)^{2}-\left[\tilde{\kappa_{1}}+\tilde{\kappa_{2}}+\tilde{\tau}\right](1-\zeta)\right] \text {, } \\
& \underline{D_{3}}=\left[\left[\tilde{\kappa_{1}}+\tilde{\sigma}\right](\zeta-1)-\left[\tilde{\beta}_{1}+\tilde{\beta}_{2}+\tilde{\beta}_{3}+\tilde{\delta}_{I}+\tilde{\tau}\right](\zeta-1)\right] \text {, } \\
& \overline{D_{3}}=\left[\left[\tilde{\kappa_{1}}+\tilde{\sigma}\right](1-\zeta)-\left[\tilde{\beta}_{1}+\tilde{\beta}_{2}+\tilde{\beta}_{3}+\tilde{\delta}_{I}+\tilde{\tau}\right](1-\zeta)\right] \text {, } \\
& \underline{D_{4}}=\left[\tilde{\kappa_{2}}(\zeta-1)-\left[\tilde{\gamma_{1}}+\tilde{\gamma_{2}}+\tilde{\sigma}+\tilde{\tau}\right](\zeta-1)\right] \text {, } \\
& \overline{D_{4}}=\left[\tilde{\kappa_{2}}(1-\zeta)-\left[\tilde{\gamma_{1}}+\tilde{\gamma_{2}}+\tilde{\sigma}+\tilde{\tau}\right](1-\zeta)\right] \text {, } \\
& D_{5}=\left[\left[\tilde{\beta}_{2}+\tilde{\gamma_{1}}\right](\zeta-1)-\left[\tilde{v_{1}}+\tilde{v_{2}}+\tilde{\delta}_{Q}+\tilde{\tau}\right](\zeta-1)\right] \text {, } \\
& \overline{D_{5}}=\left[\left[\tilde{\beta_{2}}+\tilde{\gamma_{1}}\right](1-\zeta)-\left[\tilde{v_{1}}+\tilde{v_{2}}+\tilde{\delta}_{Q}+\tilde{\tau}\right](1-\zeta)\right] \text {, } \\
& D_{6}=\left[\left[\tilde{\beta}_{1}+\tilde{v}_{1}\right](\zeta-1)-\left[\tilde{\vartheta}+\tilde{\delta}_{H}+\tilde{\tau}\right](\zeta-1)\right] \text {, } \\
& \overline{D_{6}}=\left[\left[\tilde{\beta}_{1}+\tilde{\nu}_{1}\right](1-\zeta)-\left[\tilde{\vartheta}+\tilde{\delta}_{H}+\tilde{\tau}\right](1-\zeta)\right] \text {, } \\
& \underline{D_{7}}=\left[\left[\tilde{\beta}_{3}+\tilde{\gamma_{2}}+\tilde{v_{2}}+\tilde{\vartheta}\right](\zeta-1)-\tilde{\tau}(\zeta-1)\right] \text {, } \\
& \overline{D_{7}}=\left[\left[\tilde{\beta_{3}}+\tilde{\gamma_{2}}+\tilde{\nu_{2}}+\tilde{\vartheta}\right](1-\zeta)-\tilde{\tau}(1-\zeta)\right] \text {. }
\end{aligned}
$$

Now, the second term of the series can be written as:

$$
\begin{aligned}
& \underline{S}_{1}(t, \zeta)=\frac{D_{1}}{\Gamma(y+1)}, \quad \bar{S}_{1}(t, \zeta)=\overline{D_{1}} \frac{t^{y}}{\Gamma(y+1)}, \\
& \underline{E}_{1}(t, \zeta)=\frac{D_{2}}{\Gamma(y+1)} \bar{E}_{1}(t, \zeta)=\overline{D_{2}} \frac{t^{y}}{\Gamma(y+1)}, \\
& \underline{I}_{1}(t, \zeta)=\underline{D_{3}} \frac{t^{y}}{\Gamma(y+1)}, \quad \bar{I}_{1}(t, \zeta)=\overline{D_{3}} \frac{t^{y}}{\Gamma(y+1)},
\end{aligned}
$$



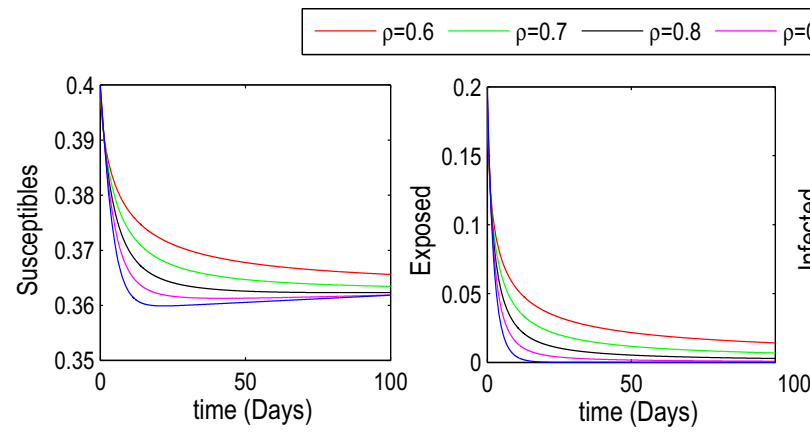

$\rho=0.9-\rho=1$
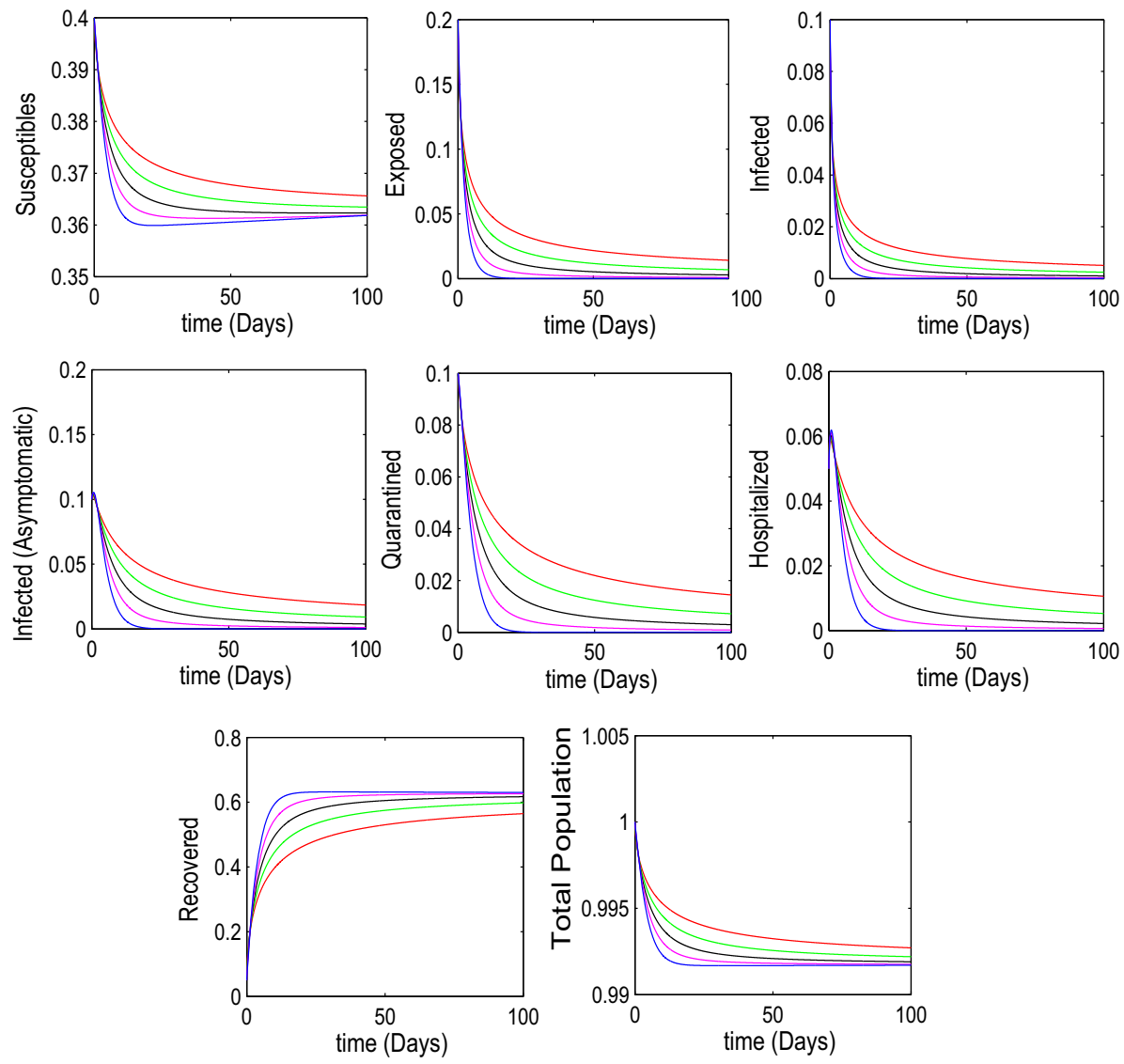

Fig. 2 Plots of state variables for different fractional orders

$$
\begin{aligned}
& \underline{A}_{1}(t, \zeta)=\frac{D_{4}}{\Gamma(y+1)}, \quad \bar{A}_{1}(t, \zeta)=\overline{D_{4}} \frac{t^{y}}{\Gamma(y+1)}, \\
& \underline{Q}_{1}(t, \zeta)=\underline{D_{5}} \frac{t^{y}}{\Gamma(y+1)}, \quad \bar{Q}_{1}(t, \zeta)=\overline{D_{5}} \frac{t^{y}}{\Gamma(y+1)}, \\
& \underline{H}_{1}(t, \zeta)=\underline{D_{6}} \frac{t^{y}}{\Gamma(y+1)}, \quad \bar{H}_{1}(t, \zeta)=\overline{D_{6}} \frac{t^{y}}{\Gamma(y+1)}, \\
& \underline{R}_{1}(t, \zeta)=\underline{D_{7}} \frac{t^{y}}{\Gamma(y+1)}, \quad \bar{R}_{1}(t, \zeta)=\overline{D_{7}} \frac{t^{y}}{\Gamma(y+1)} .
\end{aligned}
$$

Similarly, we can compute several terms of the series solution. Now, we demonstrate our results. The graph in Fig. 2 shows dynamical behavior of state variables for different values of fractional order $\rho$. From the Fig. 2, we observe that susceptible individuals decreases as the value of order of fractional derivative $\rho$ increases and also we notice that all the other state variables behave the same, i.e., $E(t), I(t), A(t), Q(t), H(t)$ and vice versa for the recovered individuals, i.e., Recovered individuals increase as the value of order of fractional derivative $\rho$ increases. Recovered individuals increase because infected people are put into quarantine 


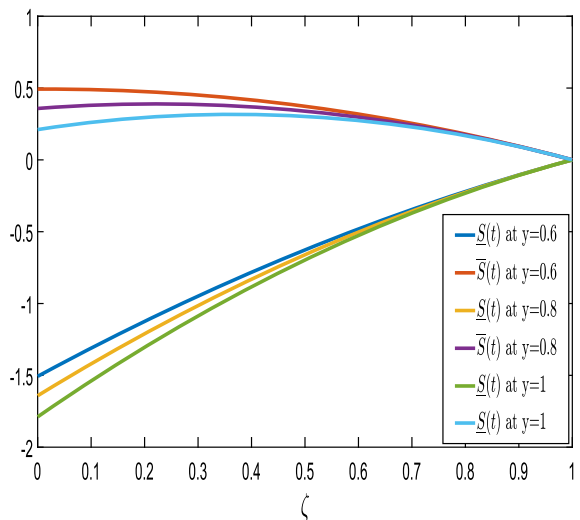

(a)

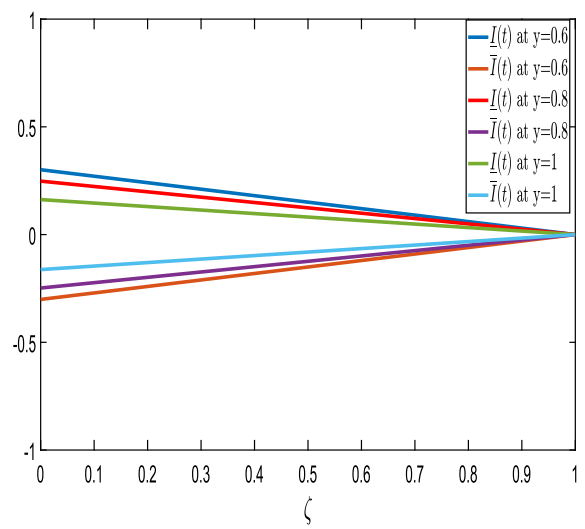

(c)



(b)

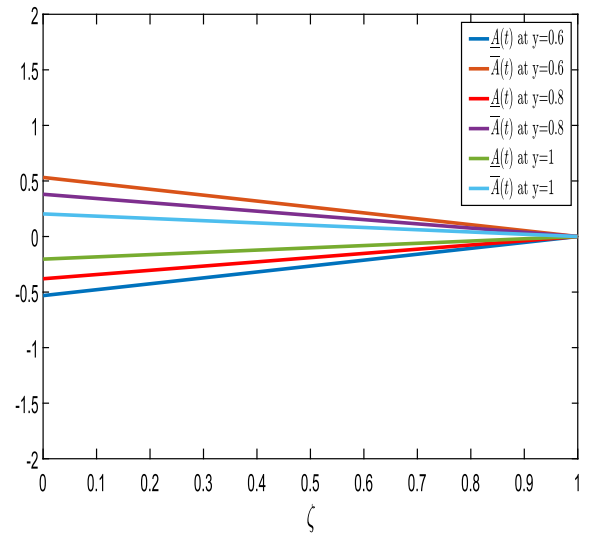

(d)

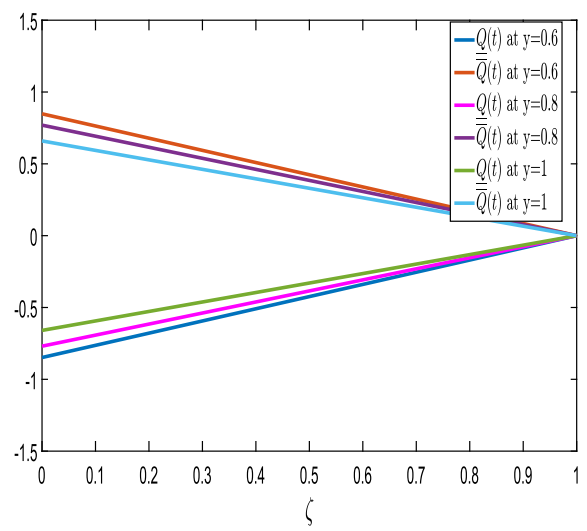

(e)

Fig. 3 Graphical representation of fuzzy solution 


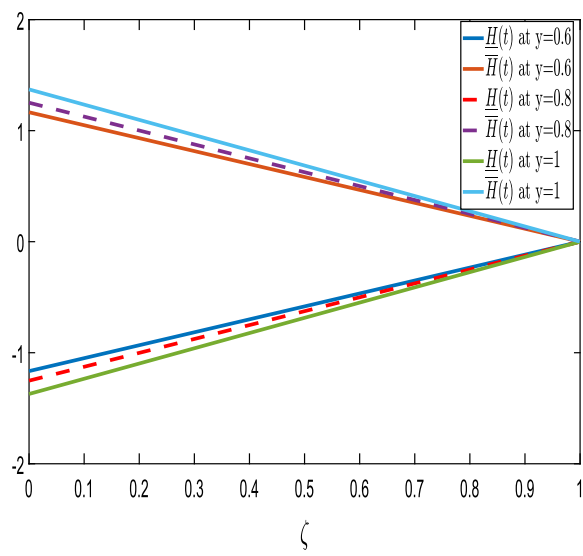

(a)

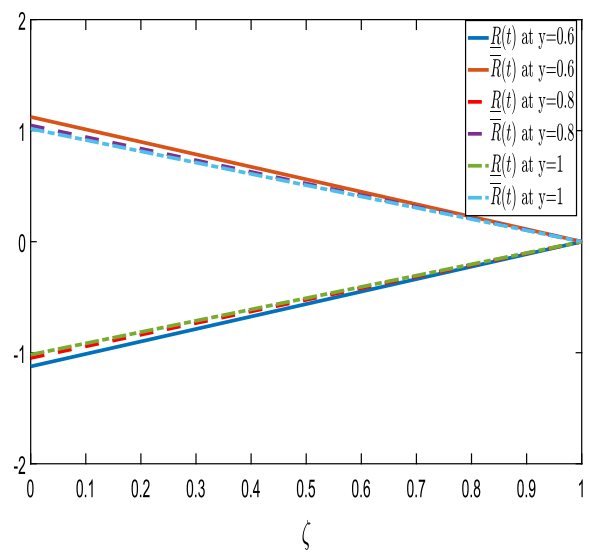

(b)

Fig. 4 Graphical representation of fuzzy solution

for 14 days. A proper medication is provided to the infected person, which kill virus in the body of Covid-19 patient. We notice the effect of fractional order on transmission of the Covid-19 disease. Variation in the fractional order causes the faster and slower growth and decay process. As the fractional order $\rho$ increases from 0.7 to 1 , the increase or decrease in the different classes becomes slower and vice versa. In Figs. 3 and 4, we simulate the obtained results of the proposed model in fuzzy case. We see that the obtained numerical results are fuzzy triangular functions. Variation in fractional order $\rho$ also effects the dynamics of the fuzzy model. When the fractional order increases from 0.6 to 1 , the diameter of the fuzzy solution increases or decreases. When $\rho=1$, then the solution curves converge to the curves of integer order model. Thus, our proposed model is more generalized than the classical integer order model.

\section{Conclusion}

In this paper, we have proposed a new fractional order model of transmission of Covid-19 with uncertainty in initial data. We have shown the positivity of state variables representing different compartments of the model. Using Gronwall inequality, we have verified that the solution of the model is bounded. Using the notion of the next-generation matrix, we have deduced the basic reproduction number $R_{o}$ of the model. We have presented the local and global stabilities of the equilibrium points by using the concept of Castillo-Chavez and Lyapunov theory with the Lasalle invariant principle, respectively. We have provided the results that demonstrate the existence and uniqueness of the solution of the considered model through the fixed point theorem of Schauder and Banach. By using the fuzzy hybrid LT, numerical results have been obtained for the compartments of the considered fuzzy fractional model (3). The results have been presented geometrically for both classical fractional order model and fuzzy model to show the transmission of the Covid-19. In the future, the current model will be investigated by introducing control factors that will control the transmission of Covid-19. 


\section{References}

1. A.Zeb, E. Alzahrani, V. S. Erturk, G. Zaman, Mathematical Model for Coronavirus Disease 2019 (COVID19) Containing Isolation Class, Biomed Res. Int., 2020, (2020) Article ID 3452402

2. P. Riyapan, S. E. Shuaib, A. Intarasit, A Mathematical Model of COVID-19 Pandemic: A Case Study of Bangkok, Thailand, Comput. Math. Methods Med., 2021, (2021) Article ID 6664483

3. I. Ahmed, G.U. Modu, A. Yusuf, P. Kumam, I. Yusuf, A mathematical model of Coronavirus Disease (COVID-19) containing asymptomatic and symptomatic classes. Results Phys. 21, 103776 (2021)

4. T. Dbouka, D. Drikakis, Fluid dynamics and epidemiology: Seasonality and transmission dynamics. Phys. Fluids 33, 021901 (2021)

5. T. Dbouka, D. Drikakis, On airborne virus transmission in elevators and confined spaces. Phys. Fluids 33, 011905 (2021)

6. T. Dbouka, D. Drikakis, Weather impact on airborne corona virus survival. Phys. Fluids 32, 093312 (2020)

7. T. Dbouka, D. Drikakis, On respiratory droplets and face masks. Phys. Fluids 32, 063303 (2020)

8. T. Dbouka, D. Drikakis, On coughing and airborne droplet transmission to humans. Phys. Fluids 32, 053310 (2020)

9. K.K. Nisar, S. Ahmad, A. Ullah, K. Shah, H. Alrabaiah, M. Arfan, Mathematical analysis of SIRD model of COVID-19 with Caputo fractional derivative based on real data. Results Phys. 21, 103772 (2021)

10. S. Rashid, Z. Hammouch, H. Aydi, A.G. Ahmad, A.M. Alsharif, Novel Computations of the TimeFractional Fisher's Model via Generalized Fractional Integral Operators by Means of the Elzaki Transform. Fractal Fract. 5(3), 94 (2021)

11. A. Traore, N. Sene, Model of economic growth in the context of fractional derivative. Alex. Eng. J. 59(6), 4843-4850 (2020)

12. S. Qureshi, A. Yusuf, A.A. Shaikh, M. Inc, D. Baleanu, Fractional modeling of blood ethanol concentration system with real data application. Chaos 29, 013143 (2019)

13. L.A. Zadeh, Fuzzy sets. Inf. Control 8, 338-353 (1965)

14. O. Kaleva, Fuzzy differential equations. Fuzzy Sets Syst. 24, 301-317 (1987)

15. V. Lupulescu, Fractional calculus for interval-valued functions. Fuzzy Sets Syst. 265, 63-85 (2015)

16. S. Arshad, V. Luplescu, Fractional differential equation with fuzzy initial condition. Electron. J. Differ. Equ. 2011, 34 (2011)

17. A. Armand, T. Allahviranloo, S. Abbasbandy, Z. Gouyandeh, Fractional relaxation-oscillation differential equations via fuzzy varaitional iteration method. J. Intell. Fuzzy Syst. 32, 363-371 (2017)

18. S. Ahmad, A. Ullah, A. Ullah, A. Akgül, T. Abdeljawad, Computational analysis of fuzzy fractional order non-dimensional Fisher equation. Phys. Scr. 96(8), 084004 (2021)

19. S. Ahmad, A. Ullah, A. Akgül, T. Abdeljawad, Semianalytical solutions of the 3rd order fuzzy dispersive partial differential equations under fractional operators. Alex. Eng. J. 60(6), 5861-5878 (2021)

20. A.A. Alderremy, J.F. Gómez-Aguilar, S. Aly, K.M. Saad, A fuzzy fractional model of coronavirus (COVID-19) and its study with Legendre spectral method. Results Phys. 21, 103773 (2021)

21. S. Ahmad, A. Ullah, K. Shah, S. Salahshour, A. Ahmadian, T. Ciano, Fuzzy fractional-order model of the novel coronavirus. Adv. Differ. Equ. 2020(1), 472 (2020). https://doi.org/10.1186/s13662-020-02934-0

22. H.J. Zimmermann, Fuzzy Set Theory and Its Applications (Kluwer Academic, Dordrecht, 1991)

23. S. Salahshour, T. Allahviranloo, S. Abbasbandy, Solving fuzzy fractional differential equations by fuzzy Laplace transform. Commun. Nonlinear Sci. Numer. Simul. 17, 1372-1381 (2012)

24. T. Allahviranloo, Fuzzy Fractional Differential Operators and Equations. Studies in Fuzziness and Soft Computing 397 (2021) 